\title{
Pre-peak ram pressure stripping in the Virgo cluster spiral galaxy NGC 4501
}

\author{
B. Vollmer ${ }^{1}$, M. Soida ${ }^{2}$, A. Chung ${ }^{3}$, J. H. van Gorkom ${ }^{4}$, K. Otmianowska-Mazur ${ }^{2}$, R. Beck ${ }^{5}$, \\ M. Urbanik ${ }^{2}$, and J. D. P. Kenney ${ }^{6}$
}

${ }^{1}$ CDS, Observatoire astronomique de Strasbourg, 11, rue de l'université, 67000 Strasbourg, France e-mail: bvollmer@astro.u-strasbg.fr

2 Astronomical Observatory, Jagiellonian University, Orla 171, 30-244 Kraków, Poland

3 NRAO Jansky fellow at University of Massachusetts, Amherst, MA 01003, USA

4 Department of Astronomy, Columbia University, 538 West 120th Street, New York, NY 10027, USA

5 Max-Planck-Insitut für Radioastronomie, Auf dem Hügel 69, 53121 Bonn, Germany

${ }^{6}$ Yale University Astronomy Department, PO Box 208101, New Haven, CT 06520-8101, USA

Received 22 June 2007 / Accepted 25 January 2008

\section{ABSTRACT}

\begin{abstract}
VIVA HI observations of the Virgo spiral galaxy NGC 4501 are presented. The HI disk is sharply truncated to the southwest, well within the stellar disk. A region of low surface-density gas, which is more extended than the main Hi disk, is discovered northeast of the galaxy center. These data are compared to existing $6 \mathrm{~cm}$ polarized radio continuum emission, $\mathrm{H} \alpha$, and optical broad band images. We observe a coincidence between the western HI and polarized emission edges, on the one hand, and a faint $\mathrm{H} \alpha$ emission ridge, on the other. The polarized emission maxima are located within the gaps between the spiral arms and the faint $\mathrm{H} \alpha$ ridge. Based on the comparison of these observations with a sample of dynamical simulations with different values for maximum ram pressure and different inclination angles between the disk and the orbital plane, we conclude that ram pressure stripping can account for the main observed characteristics. NGC 4501 is stripped nearly edge-on, is heading southwest, and is $~ 200-300$ Myr before peak ram pressure, i.e. its closest approach to M 87. The southwestern ridge of enhanced gas surface density and enhanced polarized radio-continuum emission is due to ram pressure compression. It is argued that the faint western $\mathrm{H} \alpha$ emission ridge is induced by nearly edge-on ram pressure stripping. NGC 4501 represents an especially clear example of early stage ram pressure stripping of a large cluster-spiral galaxy.
\end{abstract}

Key words. galaxies: individual: NGC 4501 - galaxies: interactions - galaxies: ISM - galaxies: kinematics and dynamics

\section{Introduction}

The Virgo cluster is dynamically young and spiral-rich making it an ideal laboratory for studying the influence of the cluster environment on spiral galaxies. Most of the Virgo spiral galaxies are HI deficient, i.e. they have lost a significant amount of their ISM (Chamaraux et al. 1980; Giovanelli \& Haynes 1983). Imaging HI observations have shown that these galaxies have truncated Hi disks (Cayatte et al. 1990). Thus, the cluster environment changes the HI content and morphology of Virgo cluster spiral galaxies. However, it remains unclear at which cluster distance the spiral galaxies lose their external Hi disk. Based on deep Effelsberg HI observations around 6 Virgo galaxies and a balance of previous detections of extraplanar gas in targeted galaxies Vollmer \& Huchtmeier (2007) propose a global picture where the outer gas disk (beyond the optical radius $R_{25}$ ) is removed much earlier than expected by the classical ram pressure criterion. They argue that the vulnerable outer disk is removed much more rapidly than predicted by the Gunn \& Gott criterion. Furthermore, Chung et al. (2007) found in a new HI imaging survey of $\sim 50$ Virgo spiral galaxies 7 galaxies with long HI tails pointing away from M 87. These galaxies are located at intermediate distances from the cluster center $(0.6-1 \mathrm{Mpc})$. They conclude that these one-sided HI tail galaxies have recently arrived in the cluster, falling in on highly radial orbits. It appears that galaxies begin to lose their gas already at intermediate distances from the cluster center through ram-pressure or turbulent viscous stripping (Nulsen 1982) and tidal interactions with neighbours, or a combination of both. The outer gas disks of spiral galaxies at projected distances smaller than $\sim 1 \mathrm{Mpc}$ are thus affected by ram pressure. In this article we show that this is also the case for the massive spiral galaxy NGC 4501 (for its properties see Table 1).

NGC 4501 is located at the lower end of the distance interval defined by Chung et al. (2007). Moreover, Cayatte et al. (1990, 1994) and Böhringer et al. (1997) proposed that it approaches the center of the Virgo cluster (M 87) on a highly eccentric orbit. NGC 4501 is moderately HI deficient and has an HI disk whose southwestern side is truncated well within optical radius $\left(R_{25}\right)$. The HI surface density distribution within the disk is asymmetric with an overdense region to the southwest showing a sharp outer edge (Cayatte et al. 1990). Since the direction of these features is pointing toward the Virgo cluster center, Cayatte et al. (1990, 1994) and Böhringer et al. (1997) speculate that they are due to ram pressure compression (Gunn \& Gott 1972). Since ram pressure only affects the interstellar medium of the galaxy and not 
Table 1. Physical parameters of NGC 4501.

\begin{tabular}{|c|c|}
\hline Other names & $\begin{array}{r}\mathrm{M} 88 \\
\mathrm{VCC} 1401 \\
\text { UGC } 7675\end{array}$ \\
\hline$\alpha(2000)^{a}$ & $12^{\mathrm{h}} 31^{\mathrm{m}} 59.2^{\mathrm{s}}$ \\
\hline$\delta(2000)^{a}$ & $14^{\circ} 25^{\prime} 14^{\prime \prime}$ \\
\hline Morphological type $^{a}$ & $\mathrm{Sb}$ \\
\hline Distance to the cluster center & $2.0^{\circ}, 0.6 \mathrm{Mpc}$ \\
\hline Optical diameter $\mathrm{D}_{25}{ }^{a}$ & $6.9^{\prime}, 34 \mathrm{kpc}$ \\
\hline$B_{T}^{0 a}$ & 9.86 \\
\hline Systemic heliocentric velocity ${ }^{a}\left(\mathrm{~km} \mathrm{~s}^{-1}\right)$ & $2280 \pm 4$ \\
\hline Velocity with respect to Virgo mean $\left(\mathrm{km} \mathrm{s}^{-1}\right)$ & 1130 \\
\hline Distance D (Mpc) & 17 \\
\hline $\operatorname{Vrot}_{\max }\left(\mathrm{km} \mathrm{s}^{-1}\right)$ & $300^{b}$ \\
\hline PA & $142^{\circ b}$ \\
\hline Inclination angle & $56^{\circ b}$ \\
\hline HI deficiency ${ }^{\mathrm{c}}$ & $0.56 \pm 0.2$ \\
\hline
\end{tabular}

${ }^{a}$ RC3, de Vaucouleurs et al. (1991); ${ }^{b}$ Guharthakurta et al. (1988); ${ }^{c}$ Cayatte et al. (1994).

its stars, one expects a symmetric stellar disk. However, the DSS $B$ band morphology is asymmetric, the southwestern side of the major axis being brighter than the northeastern side, rising the question if NGC 4501 is exclusively experiencing an interaction with the intracluster medium and if a past gravitational interaction can be excluded.

In this article we investigate the nature of the interaction of NGC 4501 with the cluster environment using VIVA (VLA Imaging of Virgo galaxies in Atomic gas, Chung et al. in prep.) HI data, VLA $6 \mathrm{~cm}$ polarized radio emission data, and a detailed dynamical model including MHD calculations. Whereas the HI distribution and kinematics show a distorted gas disk where some of the gas is pushed to the north-east, the polarized radio-continuum emission represents an ideal tracer of ram pressure compression at the opposite side of the disk. The comparison between observations and simulations shows that NGC 4501 is indeed approaching the cluster center. Ram pressure stripping can account for the main properties of this Virgo spiral galaxy.

We describe the HI and $6 \mathrm{~cm}$ polarized radio-continuum observations and their results in Sects. 2 and 3. Additional $\mathrm{H} \alpha$ data are discussed in Sect. 4. After a description of the dynamical model, the best fitting model and following MHD calculations are presented (Sect. 5). Our models are compared to the observational data in Sect. 6 followed by a discussion (Sect. 7) and our conclusions (Sect. 8).

\section{Observations}

\subsection{VIVA HI $21 \mathrm{~cm}$ line observations}

HI line observations were made on January 20 1999, using the C configuration of the VLA of the National Radio Astronomy Observatory $(\mathrm{NRAO})^{1}$. The total on-source time was $5.6 \mathrm{~h}$. The bandpasses of $2 \times 3.125 \mathrm{MHz}$ were centered at $2280 \mathrm{~km} \mathrm{~s}^{-1}$ configured to produce two polarizations and 63 channels with on-line Hanning smoothing, yielding a channel spacing of $10.4 \mathrm{~km} \mathrm{~s}^{-1}$. The nearby phase calibrator $1252+199$ was observed every $25 \mathrm{~min}$, and $1328+307$ was used as a flux calibrator.

\footnotetext{
${ }^{1}$ NRAO is a facility of National Science Foundation operated under cooperative agreement by Associated Universities, Inc.
}

We used the NRAO Astronomical Image Processing System (AIPS) for calibration and mapping. The continuum subtraction was done using a linear fit to the visibility data of line-free channels. To make the image cube we applied a weighting scheme intermediate between uniform and natural, but closer to a natural weighting scheme (robust $=1$ ), which resulted in a resolution of 17". To maximize sensitivity, we made a second data cube with tapered data (resolution of $30^{\prime \prime}$ ). The resulting rms are $0.7 \mathrm{mJy}$ and $1.2 \mathrm{mJy}$ per beam and per channel, respectively, which corresponds to $2.9 / 1.5 \times 10^{19} \mathrm{~cm}^{-2}$.

\section{2. $6 \mathrm{~cm}$ polarized radio-continuum observations}

NGC 4501 was observed for 3:55 h on December 3, 2005 with the Very Large Array (VLA) of the National Radio Astronomy Observatory (NRAO) in the D array configuration. The band passes were $2 \times 50 \mathrm{MHz}$. We used 3C 286 as the flux calibrator and $1254+116$ as the phase calibrator, the latter of which was observed every $40 \mathrm{~min}$. Maps were made for both wavelengths using the AIPS task IMAGR with ROBUST $=3$. The final cleaned maps were convolved to a beam size of $18^{\prime \prime} \times 18^{\prime \prime}$. We ended up with an rms level of the linear polarization, taken to be the mean $\mathrm{rms}$ in Stokes $Q$ and $U$, of $11 \mu \mathrm{Jy} / \mathrm{beam}$. The distribution of $6 \mathrm{~cm}$ polarized continuum emission was already published in Vollmer et al. (2007). For each map point we determined the apparent polarization $\boldsymbol{B}$-vector, defined as the $\boldsymbol{E}$-vector rotated by $90^{\circ}$. Taking into account the possible bias due to Faraday rotation (Weżgowiec et al. 2007) this represents a good approximation of the orientation of the sky-projected mean regular magnetic field, accurate to about $\pm 10^{\circ}$.

\section{Results}

The $30^{\prime \prime}$ resolution HI distribution, velocity field, and velocity dispersion distribution are shown in Fig. 1. The superposition of the HI distribution on a $B$ band and an $H$ band $(1.65 \mu \mathrm{m})$ image are presented in Figs. 2 and 3. As observed by Cayatte et al. (1990) the HI distribution shows a central hole and is truncated near the optical radius $R_{25}$. The HI surface density distribution within the disk is asymmetric with a high surface-density ridge in the southwest. The average HI surface density in the northeastern part of the disk is $\sim 3$ times lower than that of the southwestern ridge. We observe low surface-density HI $(\sim 2-5 \times$ $10^{19} \mathrm{~cm}^{-2}$ ) to the northeast of the galaxy center. This gas was not detected by Cayatte et al. (1990).

The velocity field of NGC 4501 is relatively symmetric parallel to the minor axis even in the low surface-density northeastern region. The rotation curve becomes flat at a smaller galactocentric radius in the southeast. The velocity dispersion is symmetric with two maxima caused by beam smearing over the inner part of the galaxy with a steeply rising rotation curve.

The comparison between the HI surface density distribution and optical images (Figs. 2 and 3) shows that the southwestern part of the HI disk is sharply truncated to well within the stellar disk. The outermost HI disk follows one isophotal contour in the southwest, but extends well beyond this contour in the northeast. This is also the case for a small $\left(\sim 20^{\prime \prime}\right)$ straight low surface-density region in the north pointing to the northwest. As already mentioned in Sect. 1, NGC 4501 has an asymmetric surface brightness distribution in the $B$ band with flocculent spiral arms showing pitch angles which are larger in the northeastern part of the galactic disk than in the southwestern part. In addition, the southwestern part of the disk is brighter than the northeastern part. However, the last $B$ band isophot contour 

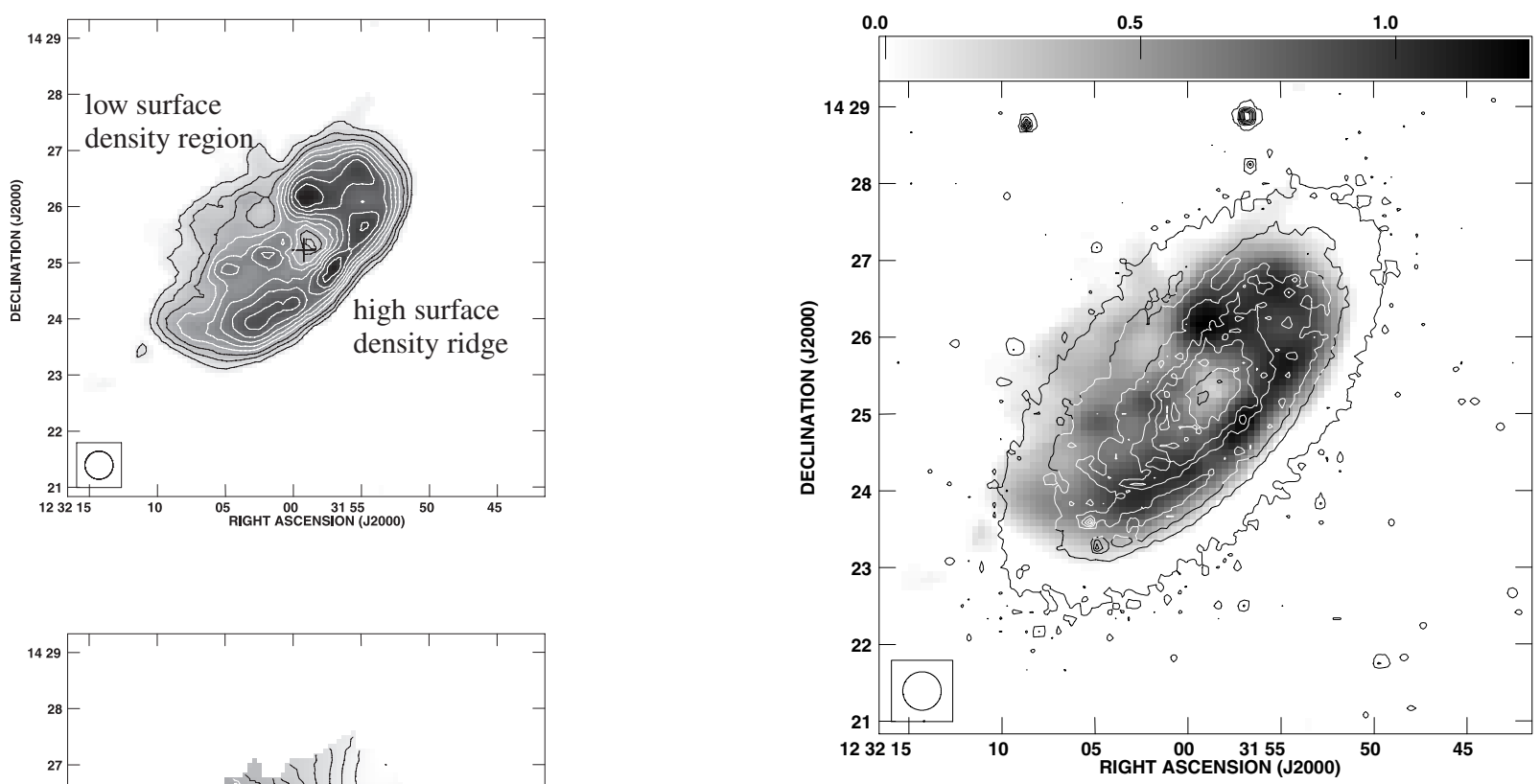

Fig. 2. Greyscale: HI surface density distribution $(0$ to $1.25 \mathrm{Jy} /$ beam $\mathrm{km} \mathrm{s}^{-1}$; resolution: $\left.30^{\prime \prime}\right)$. Contours: $B$ band image from GOLD Mine (Gavazzi et al. 2003).

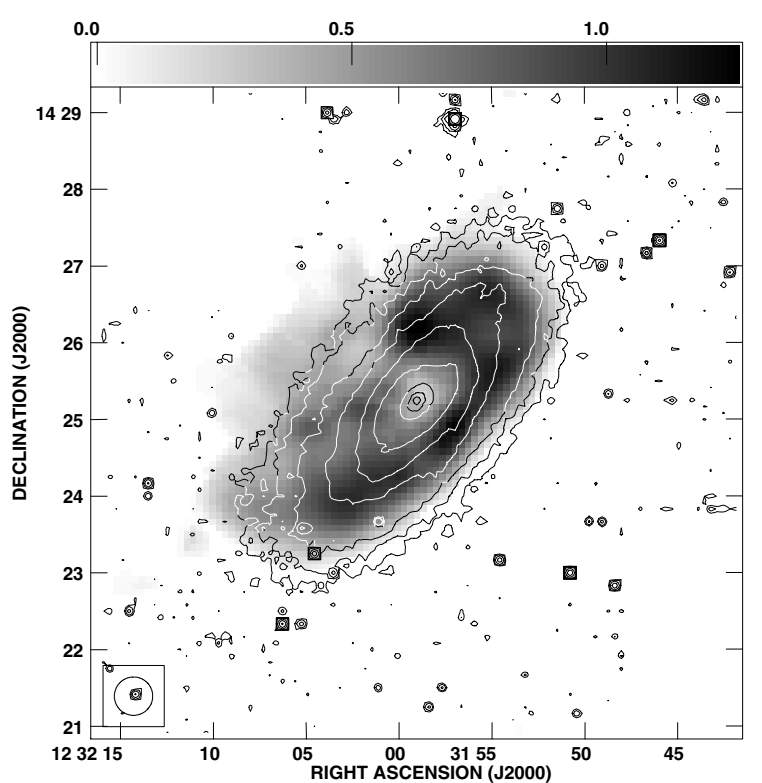

Fig. 1. HI observations of NGC 4501. Upper panel: surface density distribution. Contour levels are $(1,2,3,4,5,6,7,8,9,10) \times$ $\left(0.125 \mathrm{Jy} /\right.$ beam $\mathrm{km} \mathrm{s}^{-1}$ or $\left.1.5 \times 10^{20} \mathrm{~cm}^{-2}\right)$. The beam $\left(30^{\prime \prime} \times 30^{\prime \prime}\right)$ is plotted in the lower left corner of the image. Middle panel: velocity field. Contours are from $2010 \mathrm{~km} \mathrm{~s}^{-1}$ (northwest) to $2590 \mathrm{~km} \mathrm{~s}^{-1}$ (southeast) in steps of $20 \mathrm{~km} \mathrm{~s}^{-1}$. Lower panel: velocity dispersion distribution. Contour levels are $(10,20,30,40,50) \mathrm{km} \mathrm{s}^{-1}$.

is symmetric. In addition, NGC 4501 has a symmetric surface brightness distribution in the $H$ band which shows the old stellar component. We thus conclude that the $B$ band asymmetries are due to the star formation history and do not reflect an asymmetry of the gravitational potential.

Fig. 3. Greyscale: HI surface density distribution $(0$ to $1.25 \mathrm{Jy} /$ beam $\mathrm{km} \mathrm{s}^{-1}$; resolution: $\left.30^{\prime \prime}\right)$. Contours: $H$ band image from GOLD Mine (Gavazzi et al. 2003).

The comparison between the $6 \mathrm{~cm}$ polarized radiocontinuum emission distribution and the $17^{\prime \prime}$ resolution HI surface density distribution is shown in Fig. 4. The polarized emission shows an enhanced ridge south of the galaxy center. This ridge coincides with the high column density $\mathrm{HI}$ ridge and is located entirely within the HI gas distribution. In addition, we observe a local maximum of polarized emission $\sim 20^{\prime \prime}$ to the north of the galaxy center. In normal field spirals the polarized radiocontinuum emission is found in interarm regions, with $\boldsymbol{B}$-vectors parallel to the adjacent spiral arms (Beck 2005). As this inner 


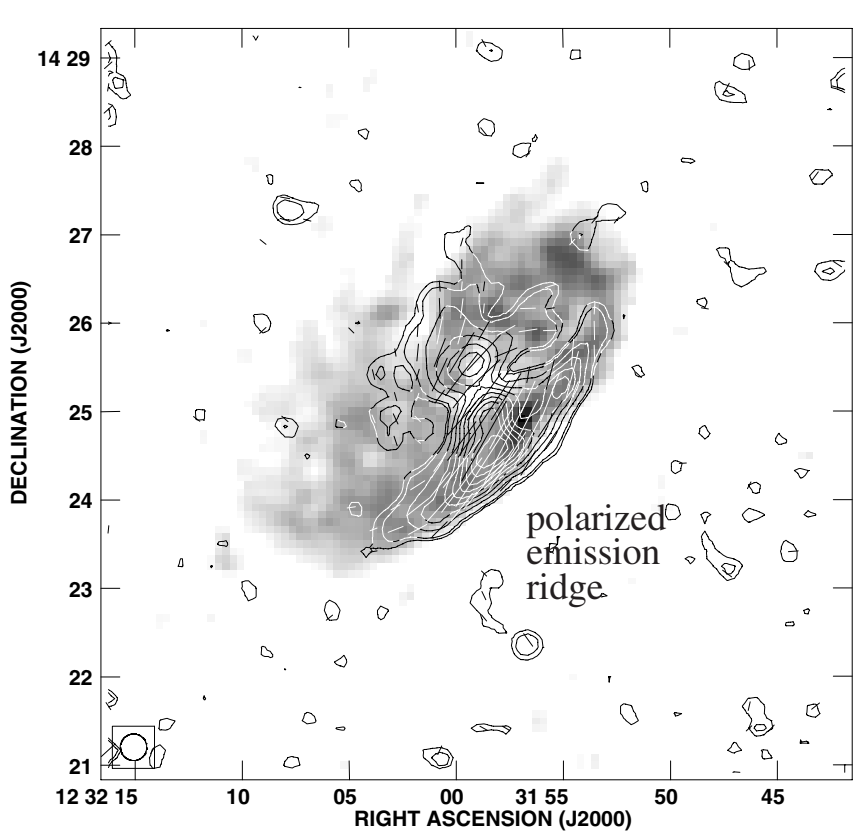

Fig. 4. Greyscale: HI surface density distribution $(0$ to $0.65 \mathrm{Jy} /$ beam $\mathrm{km} \mathrm{s}^{-1}$; resolution: 17"). Contours: $6 \mathrm{~cm}$ polarized radio-continuum emission (Vollmer et al. 2007). The contour levels are $(0.7,1,2,3,4,5,6,7,8,9,10) \times 72 \mu \mathrm{Jy} /$ beam. The vectors of the magnetic field are uncorrected for Faraday rotation. Their size is proportional to the intensity of the polarized emission. The beam $\left(18^{\prime \prime} \times 18^{\prime \prime}\right)$ is plotted in the lower left corner of the image.

interarm region is not resolved in our radio polarization data, the origin of the polarized emission in the north remains unclear. The magnetic field vectors are compared to the optical $B$ band images in Fig. 5. If the large-scale magnetic field is determined by the spiral arms, the magnetic field vectors should follow the spiral structure. This is what we observe in the inner $\sim 1^{\prime}=5 \mathrm{kpc}$. However, the magnetic field vectors cross the spiral arm in the most northeastern part of the polarized emission distribution. This is similar to the behavior of the largescale magnetic field in NGC 3627 (Soida et al. 2001) where a magnetic arm crosses an optical arm, which is interpreted as a sign of tidal interactions within the Leo Triplet group. On the other hand, the magnetic field within the ridge of enhanced polarized radio-continuum emission follows the spiral structure. Figure 6 shows profiles parallel to the minor axis centered on the major axis $\sim 1^{\prime}$ southeast of the galaxy center (see Fig. 5). We show the HI surface density, the $6 \mathrm{~cm}$ polarized radio-continuum emission, and the $\mathrm{H} \alpha$ emission. The HI and the polarized emission profiles rise together and peak at the same location. The polarized radio-continuum emission is enhanced by ram pressure compression of the magnetic field. If the original magnetic field was random, the compressed field is anisotropic and we do not expect Faraday rotation. A detailed analysis of Faraday rotation measures of Virgo Cluster spirals will be subject of future studies. The intensity ratio between the peak and the base of the polarized emission can be used to measure the compression ratio (Beck et al. 2005). The high resolution faint $\mathrm{H} \alpha$ emission coincides with the HI and polarized emission peaks. A similar behavior is also observed in the interacting spiral galaxy NGC 2276 (Hummel \& Beck 1995) where a ridge of enhanced polarized radio-continuum emission is observed in the south of the galaxy, in the group spiral galaxy NGC 2442 (Harnett et al. 2004) where the northern polarized emission ridge is shifted

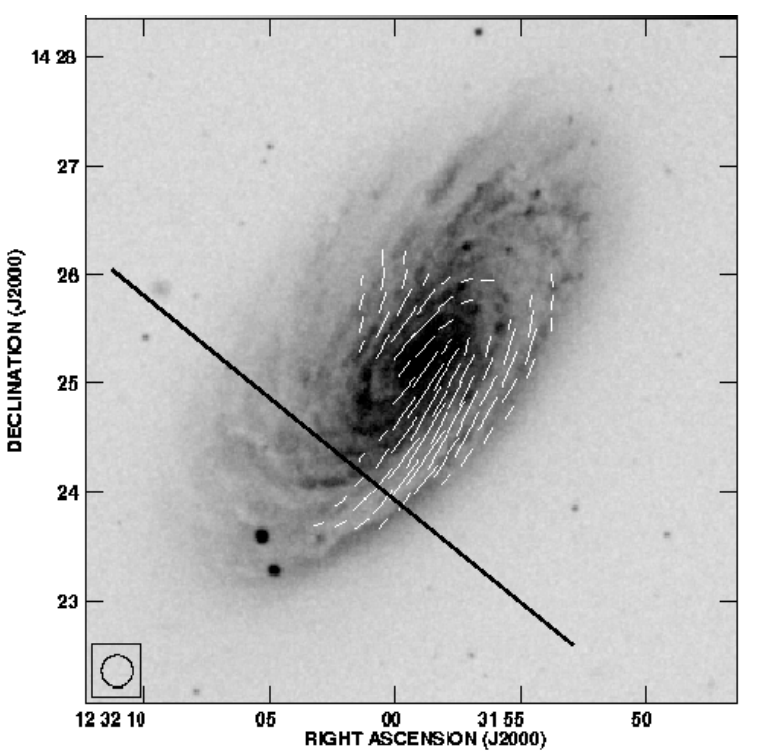

Fig. 5. Greyscale: $B$ band image from GOLD Mine (Gavazzi et al. 2003). The vectors of the magnetic field are uncorrected for Faraday rotation (resolution: 18"). Their size is proportional to the intensity of the polarized emission as in Fig. 4. The line indicates the slit position for the profile of Fig. 6 .

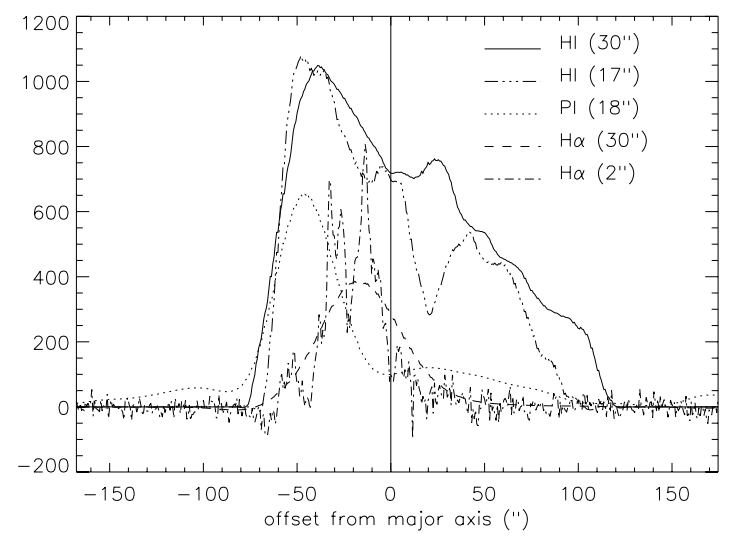

Fig. 6. Profiles parallel to the minor axis $\sim 1^{\prime}$ southeast of the galaxy center (see Fig. 5). Solid: HI surface density (resolution: 30"); dashdouble-dotted: HI surface density (resolution: 17"); dotted: $6 \mathrm{~cm}$ polarized radio-continuum emission (resolution: $18^{\prime \prime}$ ); dashed: $\mathrm{H} \alpha$ emission at a resolution of $30^{\prime \prime}$; dash-dotted: $\mathrm{H} \alpha$ emission at a $2^{\prime \prime}$ resolution. The $y$-axis has arbitrary units. Left corresponds to the southwest. The position of the major axis is marked with the vertical line.

towards the outer edge of the star-forming region, and the Virgo spiral galaxy NGC 4254 (Soida et al. 1996) where the H $\alpha$-bright spiral arm is placed inwards with respect to the compressed region. In NGC 2276 a tidal compression due to a galaxy-galaxy interaction is responsible for the enhanced polarized emission. For NGC 2442 and NGC 4254 a tidal interaction also cannot be excluded.

\section{Star formation}

We use an $\mathrm{H} \alpha$ image to compare the HI and polarized radiocontinuum emission to the distribution of massive star formation (Figs. 7 and 8). The $\mathrm{H} \alpha$ distribution shows a ring structure in the inner disk $\left(R<40^{\prime \prime}\right)$ and a prominent spiral arm in the northwest. The spiral arm begins north of the galaxy center, continues to the northwest, bends to the southwest, and finally follows 


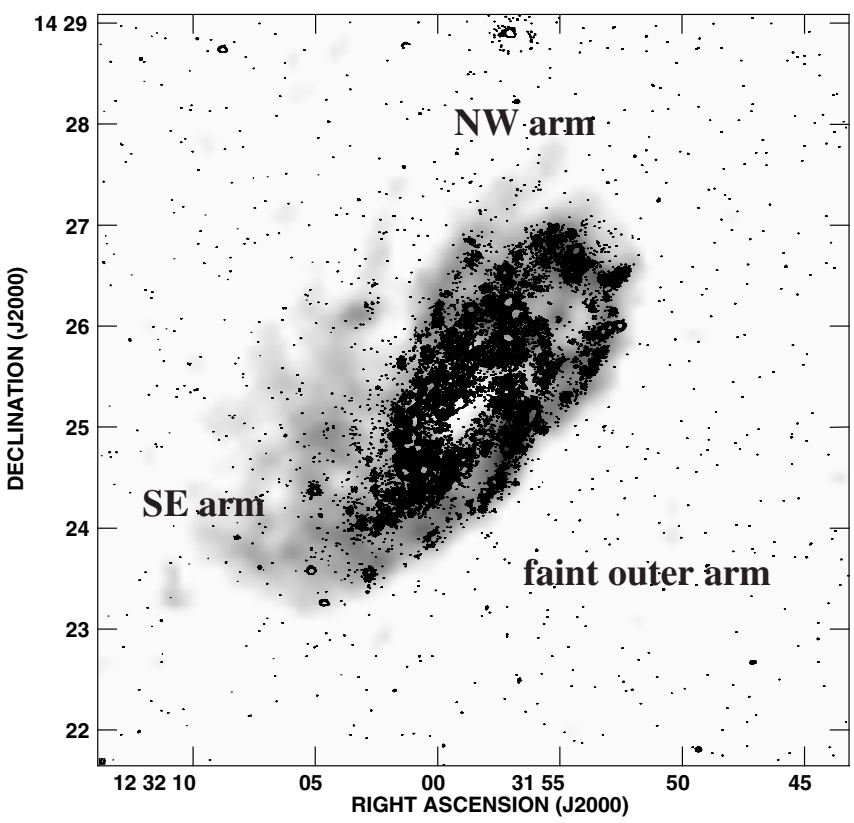

Fig. 7. Greyscale: HI surface density distribution $(0$ to $0.65 \mathrm{Jy} /$ beam $\mathrm{km} \mathrm{s}^{-1}$; 17" resolution). Contours: $\mathrm{H} \alpha$ emission distribution from GOLD Mine (Gavazzi et al. 2003). The contour levels are chosen in a way to make the faint southwestern $\mathrm{H} \alpha$ arm visible.

the southwestern edge of the HI distribution. There is the beginning of a second $\mathrm{H} \alpha$ spiral arm in the southeast of the galactic disk. This second spiral arm abruptly ends $\sim 1^{\prime}=5 \mathrm{kpc}$ from the galaxy center. The comparison between the $\mathrm{H} \alpha$ emission distribution and the $6 \mathrm{~cm}$ polarized radio-continuum emission distribution (Fig. 8) shows that the maximum of the polarized emission south of the galaxy center is located between the inner $\mathrm{H} \alpha$ ring and the outer $\mathrm{H} \alpha$ arm. The location of maximum polarized radio-continuum emission may indicate the region of strongest field compression, coinciding with maximum HI emission. Alternatively, the field could be tangled in star forming regions located in the $\mathrm{H} \alpha$ arms. Note that the limited resolution of the radio observations leads to beam depolarization in the inner disk around the major axis if the field lines follow the strong curvature of the optical spiral structure. The second local maximum northwest from the first maximum is also located in an $\mathrm{H} \alpha$ emission gap. On the other hand, the two local maxima located at $\sim 20^{\prime \prime}$ north and south of the galaxy center do not coincide with such clear gaps. The maxima in the ridge of enhanced polarized emission are thus different from those of the inner disk, which are possibly due to the shear caused by the spiral arms. Spitzer $8 \mu \mathrm{m}$ and $24 \mu \mathrm{m}$ maps (in prep.) show essentially the same spiral arm features as the $\mathrm{H} \alpha$.

\section{Models}

To interpret the observations described in Sect. 3, we compare them to a detailed dynamical model which includes ram pressure. In a second step, we solve the induction equation for the velocity fields of the dynamical model to determine the evolution of the large-scale magnetic field. Assuming a distribution of relativistic electrons, we then calculate the model distribution of polarized radio-continuum emission.

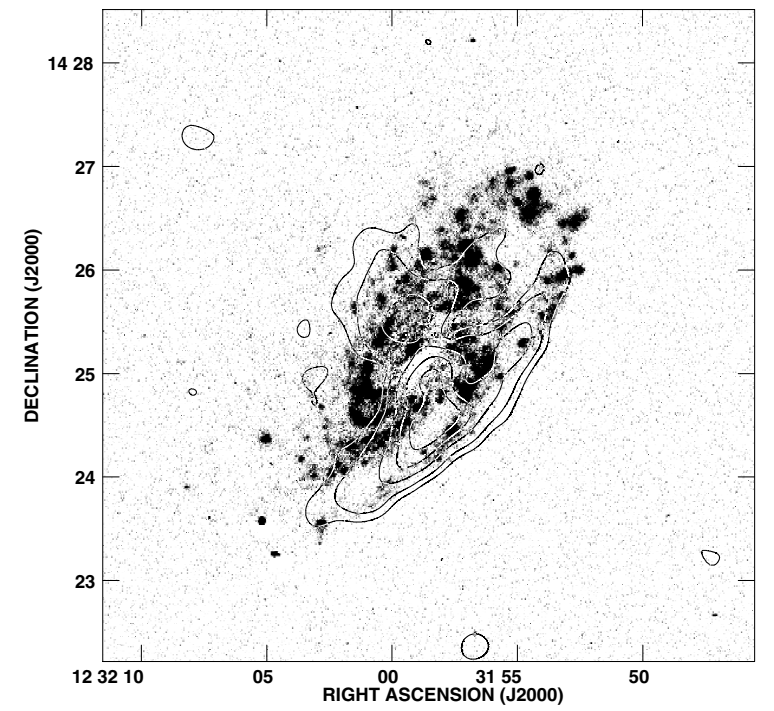

Fig. 8. Greyscale: $\mathrm{H} \alpha$ emission distribution. Contours: $6 \mathrm{~cm}$ polarized radio-continuum emission. The contour levels are $(1,2,4,6,8,10) \times$ $72 \mu \mathrm{Jy} /$ beam.

Table 2. Total mass, number of particles $N$, particle mass $M$, and smoothing length $l$ for the different galactic components.

\begin{tabular}{lllll}
\hline \hline Component & $M_{\text {tot }}\left(M_{\odot}\right)$ & $N$ & $M\left(M_{\odot}\right)$ & $l(\mathrm{pc})$ \\
\hline halo & $7.3 \times 10^{11}$ & 16384 & $4.5 \times 10^{7}$ & 1200 \\
bulge & $2.5 \times 10^{10}$ & 16384 & $1.5 \times 10^{6}$ & 180 \\
disk & $1.3 \times 10^{11}$ & 16384 & $7.9 \times 10^{5}$ & 240 \\
\hline
\end{tabular}

\subsection{Dynamical model}

We use the $N$-body code described in Vollmer et al. (2001) which consists of two components: a non-collisional component that simulates the stellar bulge/disk and the dark halo, and a collisional component that simulates the ISM.

The non-collisional component consists of 49125 particles, which simulate the galactic halo, bulge, and disk. The characteristics of the different galactic components are shown in Table 2. The resulting rotation velocity is $\sim 300 \mathrm{~km} \mathrm{~s}^{-1}$.

We have adopted a model where the ISM is simulated as a collisional component, i.e. as discrete particles which possess a mass and a radius and which can have inelastic collisions (sticky particles). Since the ISM is a turbulent and fractal medium (see e.g. Elmegreen \& Falgarone 1996), it is neither continuous nor discrete. The volume filling factor of the warm and cold phases is smaller than one. The warm neutral and ionized gas fill about $30-50 \%$ of the volume, whereas cold neutral gas has a volume filling factor smaller than $10 \%$ (Boulares \& Cox 1990). It is not clear how this fraction changes, when an external pressure is applied. In contrast to smoothed particles hydrodynamics (SPH), which is a quasi continuous approach and where the particles cannot penetrate each other, our approach allows a finite penetration length, which is given by the mass-radius relation of the particles. Both methods have their advantages and their limits. The advantage of our approach is that ram pressure can be included easily as an additional acceleration on particles that are not protected by other particles (see Vollmer et al. 2001). In this way we avoid the problem of treating the huge density contrast between the ICM $\left(n \sim 10^{-4} \mathrm{~cm}^{-3}\right)$ and the ISM $\left(n>1 \mathrm{~cm}^{-3}\right)$ of the galaxy. 
The 20000 particles of the collisional component represent gas cloud complexes which are evolving in the gravitational potential of the galaxy. The total assumed gas mass is $M_{\text {gas }}^{\text {tot }}=8.9 \times$ $10^{9} M_{\odot}$, which corresponds to the total neutral gas mass before stripping. Following Vollmer \& Huchtmeier (2007) we assume an initial gas disk which is truncated at approximately the optical radius $(\sim 17 \mathrm{kpc})$. To each particle a radius is attributed depending on its mass. During the disk evolution the particles can have inelastic collisions, the outcome of which (coalescence, mass exchange, or fragmentation) is simplified following Wiegel (1994). This results in an effective gas viscosity in the disk.

As the galaxy moves through the ICM, its gas clouds are accelerated by ram pressure. Within the galaxy's inertial system its clouds are exposed to a wind coming from a direction opposite to that of the galaxy's motion through a static ICM. The temporal ram pressure profile has the form of a Lorentzian, which is a good approximation for galaxies on highly eccentric orbits within the Virgo cluster (Vollmer et al. 2001). The effect of ram pressure on the clouds is simulated by an additional force on the clouds in the wind direction. Only clouds which are not protected by other clouds against the wind are affected.

The particle trajectories are integrated using an adaptive timestep for each particle. This method is described in Springel et al. (2001). The following criterion for an individual timestep is applied:

$\Delta t_{i}=\frac{20 \mathrm{~km} \mathrm{~s}^{-1}}{a_{i}}$

where $a_{i}$ is the acceleration of the particle $i$. The minimum value of $t_{i}$ defines the global timestep used for the Burlisch-Stoer integrator that integrates the collisional component.

\subsection{Search for the best fit model}

In this section we will constrain the parameters of the ram pressure stripping event which are (i) the peak ram pressure; (ii) the temporal ram pressure profile; (iii) time since peak ram pressure; (iv) the disk-wind angle between the galaxy's disk and the intracluster medium wind direction; and (v) the azimuthal viewing angle for the observed inclination and position angles (Fig. 9). These parameters are related to the observed quantities which are (1) the position angle PA, (2) the inclination angle of the galactic disk incl, (3) the line-of-sight velocity of the galaxy with respect to the cluster mean, and (4) the projected ICM wind direction. The position angle and inclination of NGC 4501 define the galaxy plane in three dimensional space. The model galaxy can then be rotated within this plane by the azimuthal viewing angle (see below). The three dimensional model wind direction, the line-of-sight velocity of the galaxy, and the projected ICM wind direction are thus functions of the azimuthal viewing angle.

We assume a highly eccentric galaxy orbit within the Virgo cluster leading to a temporal ram pressure profile of the form:

$p_{\text {ram }}=p_{\text {max }} \frac{t_{\mathrm{HW}}^{2}}{t^{2}+t_{\mathrm{HW}}^{2}}$,

where $t_{\mathrm{HW}}$ is the width of the profile (Vollmer et al. 2001). We define $t=0 \mathrm{Myr}$ as the time when ram pressure is maximum. As shown in Vollmer et al. (2001) the width of the profile depends on the ram pressure maximum. For a high maximum ram pressure the galaxy has to come very close to the cluster center and consequently its velocity is higher while it crosses the high ICM density region in the cluster core. We use two typical
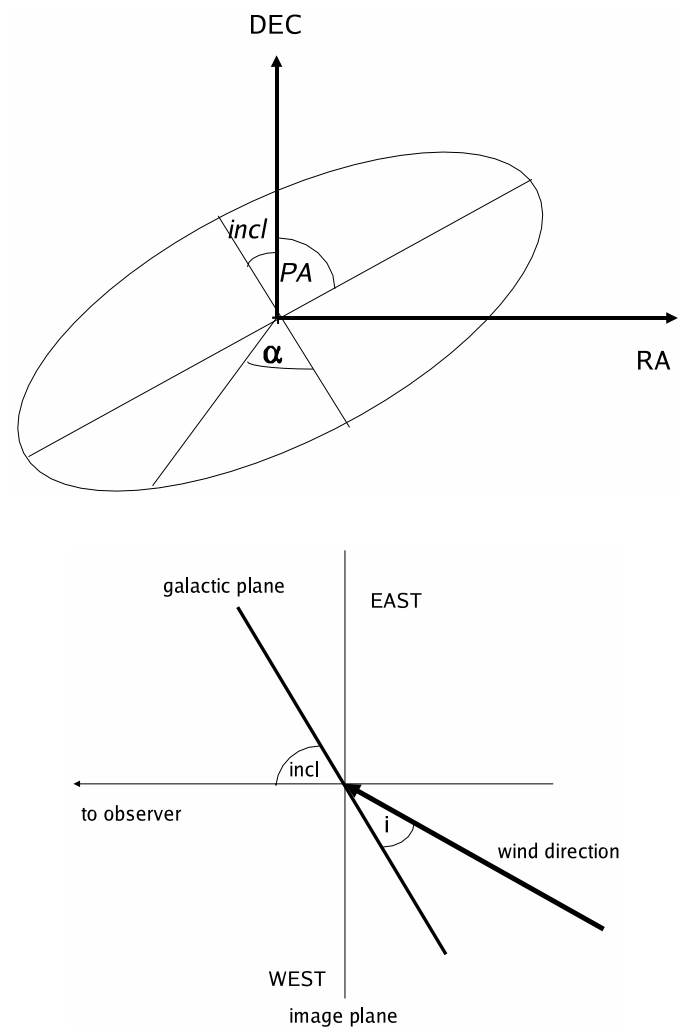

Fig. 9. Galaxy geometry. Upper panel: image plane. PA: position angle; incl: inclination of the galactic disk with respect to the plane of the sky; $\alpha$ : azimuthal viewing angle within the galaxy plane defined by PA and incl. Lower panel: perpendicular view from the top. $i$ : disk-wind angle.

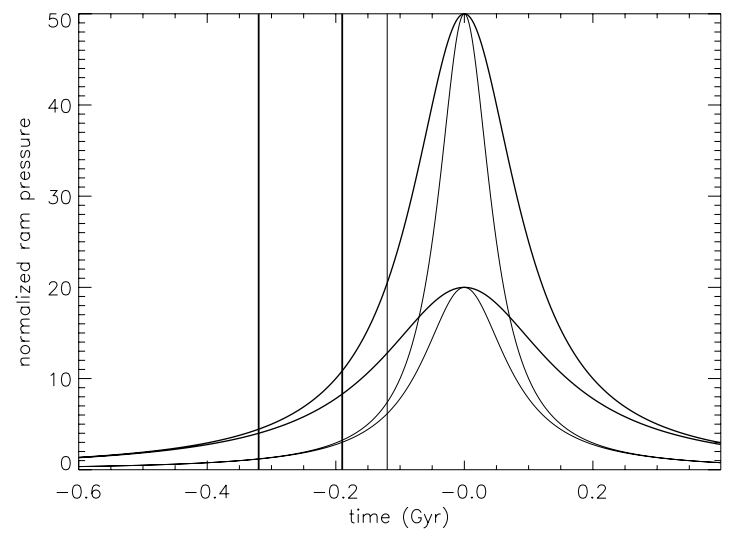

Fig. 10. The adopted temporal ram pressure profiles for the low ram pressure (LRP) and the high ram pressure (HRP) model. Thick lines: ram pressure profiles with doubled widths (LRP1/2, HRP1/2). The vertical lines show the adopted timesteps for the comparison between model and observations.

profiles for strong and moderately strong ram pressure stripping (Fig. 10, see Vollmer et al. 2001):

1. low ram pressure model (LRP): $p_{\text {max }}=2000 \mathrm{~cm}^{-3}\left(\mathrm{~km} \mathrm{~s}^{-1}\right)^{2}, t_{\mathrm{HW}}=80 \mathrm{Myr}$ and

2. high ram pressure model (HRP): $p_{\max }=5000 \mathrm{~cm}^{-3}\left(\mathrm{~km} \mathrm{~s}^{-1}\right)^{2}, t_{\mathrm{HW}}=50 \mathrm{Myr}$.

It turned out that, in both simulations, NGC 4501 encounters peak ram pressure within $120 \mathrm{Myr}$. Since this is not consistent with its location within the cluster assuming classical galaxy orbits in a static smooth intracluster medium (Vollmer et al. 2001), 

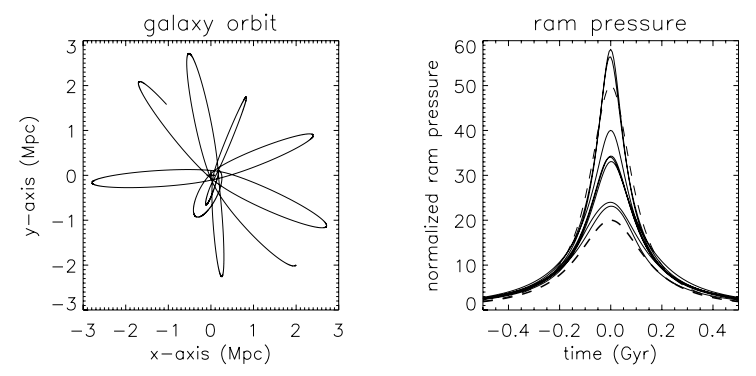

Fig. 11. Left panel: galaxy orbit in the Virgo cluster leading to temporal ram pressure profiles with a larger width. Right panel: resulting ram pressure profiles (solid lines) and LRP1/2, HRP1/2 ram pressure profiles (dashed lines).

we made a second set of simulations for which we doubled the widths of the temporal ram pressure profiles (Fig. 10):

1. low ram pressure model (LRP1/2):

$p_{\text {max }}=2000 \mathrm{~cm}^{-3}\left(\mathrm{~km} \mathrm{~s}^{-1}\right)^{2}, t_{\mathrm{HW}}=160 \mathrm{Myr}$ and

2. high ram pressure model (HRP1/2):

$p_{\max }=5000 \mathrm{~cm}^{-3}\left(\mathrm{~km} \mathrm{~s}^{-1}\right)^{2}, t_{\mathrm{HW}}=100 \mathrm{Myr}$.

In these simulations the galaxy does not go as close to the cluster core (Fig. 11) as in the LRP and HRP models. The minimum distance to the cluster center is about $10 \%$ larger than that of the orbits shown in Vollmer et al. (2001). The minimum distance of the LRP $1 / 2$ is about $0.25 \mathrm{Mpc}$, that of the HRP1/2 model is about $0.1 \mathrm{Mpc}$. The larger width of the ram pressure profile is mainly due to a broader intracluster medium distribution compared to the LRP and HRP simulations.

With these 4 simulations we aim to reproduce the main characteristics of our Hi observations:

- the low surface-density gas located northeast of the galaxy center;

- the region of high surface-density gas at the opposite windward side;

- the asymmetry along the major axis with a more extended emission to the southeast;

- the rising rotation curve to the northwest;

- the southwestern ridge of polarized radio-continuum emission.

The first snapshot selection is mainly based on the HI morphology. For the LRP and HRP simulations we identify one best fit model (LRP: Fig. 13, HRP: Fig. 14), for the LRP1/2 and HRP1/2 simulations two best fit models (LRP1 and LRP2: Fig. 15; HRP1 and HRP2: Fig. 16) are selected. In a second step (Sect. 6), gas kinematics and the polarized radio contiuum emission distribution are taken into account. Table 3 summarizes our findings.

Ram pressure efficiency depends on the disk-wind angle $i$ between the galactic disk and the ICM wind direction (Vollmer et al. 2001). A disk-wind angle of $i<-30^{\circ}$ is not compatible with NGC 4501's positive radial velocity with respect to the Virgo cluster. Disk-wind angles $i>30^{\circ}$ lead to projected wind directions to the northeast for almost all azimuthal viewing angles. However, the HI and polarized emission observations indicate that the projected wind direction has an important component to the west. Therefore, we do not consider stripping with $|i|>30^{\circ}$. For each model (LRP and HRP) we made 4 simulations with 4 different disk-wind angles between the galaxy's disk and the ICM wind direction: (i) $i=-30^{\circ}$; (ii) $i=-10^{\circ}$; (iii) $i=10^{\circ}$;
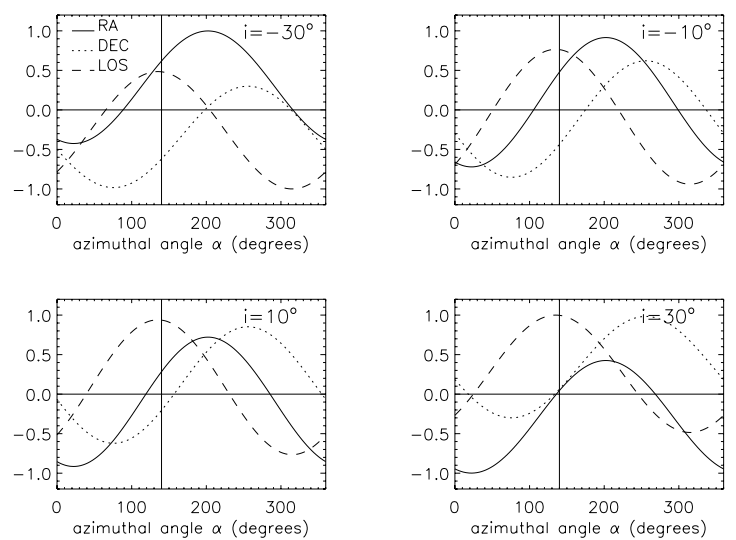

Fig. 12. Components of the 3D direction of the galaxy's velocity as a function of the azimuthal viewing angle $\alpha$. The length of the vector is unity. Solid line: right ascension; dotted line: declination; dashed line: line-of-sight direction for four different disk-wind angle between the galactic disk and the ICM wind $i=-30^{\circ}, i=-10^{\circ}, i=10^{\circ}$, and $i=30^{\circ}$. The vertical line represents a viewing angle of $140^{\circ}$.

and (iv) $i=30^{\circ}$. A disk-wind of $i=0^{\circ}$ means that the galactic disk is parallel to the ICM wind direction. The azimuthal viewing angle, which is the angle in the galactic plane defined by the galaxy's inclination and position angles, is chosen in a way to fit the observed HI distribution, i.e. the position of the extended northeastern low surface-density gas and to reproduce the positive line-of-sight component of the wind direction (the galaxy is moving away from the observer). In Figs. 12 we show the components of the 3D ICM wind direction. For the chosen azimuthal viewing angle $\alpha=140^{\circ}$ the line-of-sight velocity is positive and represents between $50 \%$ and $100 \%$ of the total velocity with respect to the cluster mean.

In Figs. 13 and 14 we show snapshots of the LRP and HRP model at 3 different timesteps $-150 \mathrm{Myr},-100 \mathrm{Myr}$, and $-50 \mathrm{Myr}$, i.e. before the ram pressure maximum. All model gas distributions at $t=-150$ Myr have symmetric outer contours and a local surface-density maximum in the southeast, which is due to the internal spiral structure. At this timestep ram pressure is not strong enough to affect the gas distribution of the disk significantly. However, gas of lower surface density is already displaced for $t<-150 \mathrm{Myr}$. At $t=-120 \mathrm{Myr}$ the southwestern edge of the gas disk is now compressed and shows a sharp edge. On the opposite side, i.e. in the northeast, gas is pushed to larger galactic radii. Since the gas is still rotating there, it forms a kind of detached low surface spiral arm. This arm is located somewhat closer to the disk for $i=30^{\circ}$ than for $i=-30^{\circ}$, because it is located above the disk which makes its projected distance smaller. At $t=-90$ Myr the northeastern low surface arm has moved to the southeast and its projected distance from the main disk has increased. The LRP and HRP models are indistinguishable at $t=-150 \mathrm{Myr}$. At $t=-120 \mathrm{Myr}$ the overall HI morphologies of the LRP and HRP model snapshots are similar and it is mainly the structure of the surface density distribution of the northeastern arm and the gas disk which change from one model to another. The latter statement also applies for the snapshots at $t=-90$ Myr. Based on these snapshots, only a simulation snapshot at $t=-120 \mathrm{Myr}$ reproduces the observed southwestern compression region and the northeastern low surface-density arm. In all model snapshots at $t=-120 \mathrm{Myr}$ we observe a region of high surface density $\sim 5 \mathrm{kpc}$ southwest of the galaxy center, which is not present in the HI data. Since this is due to the internal structure of the model galaxy, which (i) is not affected by 
Table 3. Features which are reproduced by the different models.

\begin{tabular}{lcccccc}
\hline \hline Model & LRP & HRP & LRP1 & LRP2 & HRP1 & HRP2 \\
\hline NE low surface density gas & + & + & + & + & + & + \\
SW high surface density gas ridge & + & + & + & + & + & + \\
HI asymmetry along major axis & - & - & - & + & - & + \\
NW rising rotation curve & + & + & + & + & + & + \\
SW ridge of polarized radio emission & + & + & + & + & + & + \\
symmetric velocity dispersion distribution & + & + & + & - & + & - \\
consistency with galaxy orbit & - & - & + & $?$ & + & $?$ \\
\hline
\end{tabular}
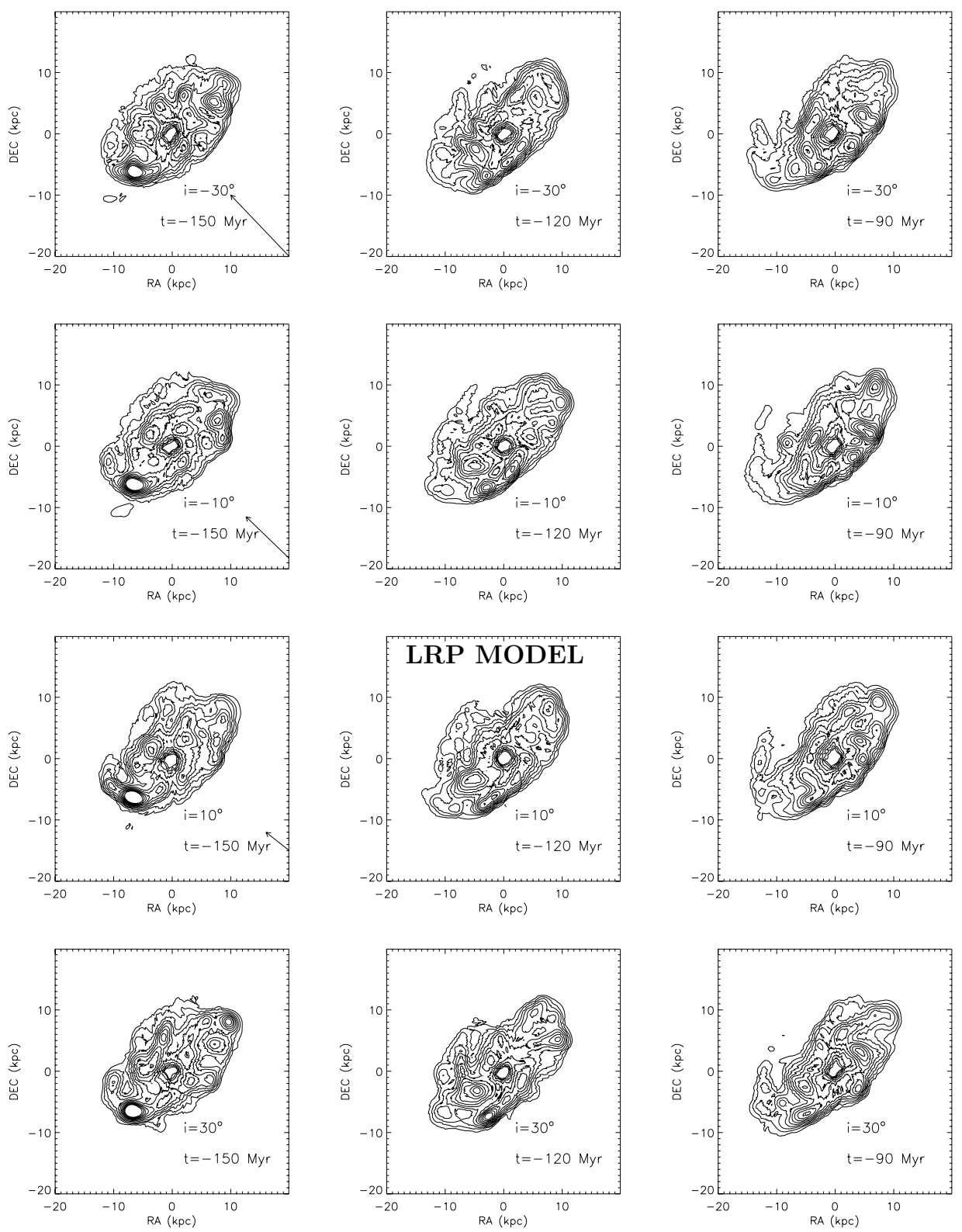

Fig. 13. Model snapshots of the gas column density for different simulations of the low ram pressure (LRP) model. Contours are (1, 2, 3, 4, 5, $6,7,8,9,10) \times 4 \times 10^{20} \mathrm{~cm}^{-2}$. The ram pressure profile for all simulations isq given by Eq. (2). The disk-wind angle $i$ between the orbital and the galaxy's disk plane and the timestep of the snapshot are varied. Left column: $t=-150$ Myr. Middle column: $t=-100$ Myr. Right column: $t=-50$ Myr. Upper row: $i=-30^{\circ}$. Middle rows: $i=-10^{\circ}$ and $i=10^{\circ}$. Lower row: $i=30^{\circ}$. The arrow represents the projected ICM wind direction. The absence of the arrow for $i=30^{\circ}$ is due to a zero projected galaxy velocity.

ram pressure because of its location in the inner galactic disk, and (ii) depends on the initial conditions, we do not take it into account for the comparison between models and observations. Based on a detailed comparison of the model gas distribution, including the southwestern overdensities and the northeastern low surface-density arm, we chose two final snapshots with $i=10^{\circ}$ for the LRP model and $i=-10^{\circ}$ for the HRP model labeled in Figs. 13 and 14. 

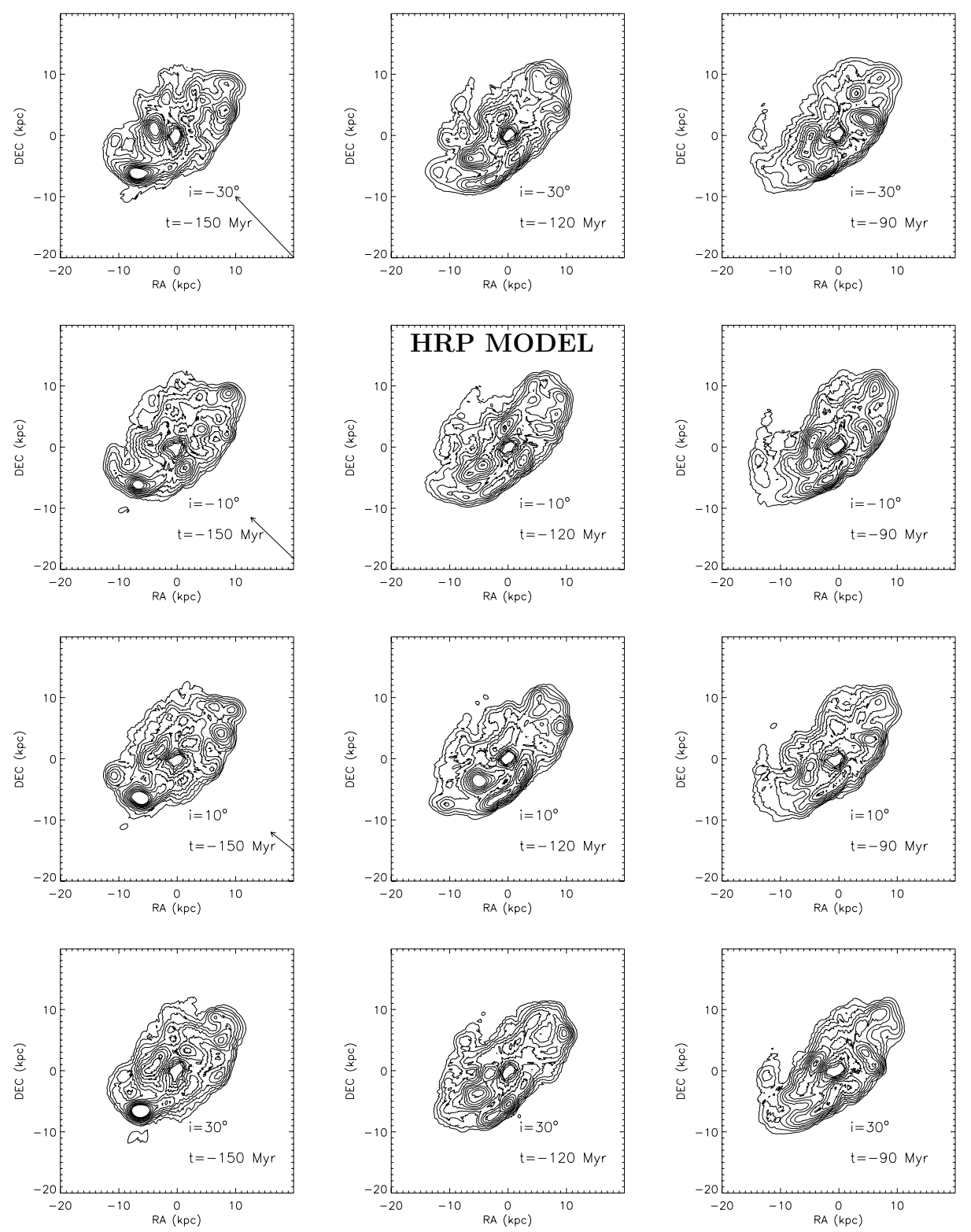

Fig. 14. Model snapshots of the gas column density for different simulations of the high ram pressure (HRP) model. Contours are the same as in Fig. 13. The ram pressure profile for all simulations is given by Eq. (2). The disk-wind angle $i$ between the orbital and the galaxy's disk plane and the timestep of the snapshot are varied. Left column: $t=-150$ Myr. Middle column: $t=-100$ Myr. Right column: $t=-50$ Myr. Upper row: $i=-30^{\circ}$. Middle rows: $i=-10^{\circ}$ and $i=10^{\circ}$. Lower row: $i=30^{\circ}$. The absence of the arrow for $i=30^{\circ}$ is due to a zero projected galaxy velocity.

Both simulations yield a timescale of 120 Myr before peak stripping. The galaxy is moving to the southwest with a velocity component in the sky-plane of $\sim 1000 \mathrm{~km} \mathrm{~s}^{-1}$. Until peak ram pressure it will cover a distance of $0.13 \mathrm{Mpc}$. Its actual distance from the cluster center at peak ram pressure will be greater than $0.5 \mathrm{Mpc}$. However, typical orbits associated to the ram pressure profiles LRP and HRP have impact parameters of 0.1-0.2 Mpc (Vollmer et al. 2001). To reconcile this difference, we doubled the widths of the temporal ram pressure profiles (LRP1/2 and HRP1/2 simulations; Fig. 10). This is still within the uncertainty of the intracluster medium density distribution given by Schindler et al. (1999). The impact parameters for orbits associated with these ram pressure profiles are $0.2-0.4 \mathrm{Mpc}$. Since $i=30^{\circ}$ lead to negligible velocity components in the plane of the sky for the adopted azimuthal viewing angle (Fig. 12), we do not consider this possibility anymore.
In Figs. 15 and 16 we show snapshots of the LRP1/2 and HRP1/2 model at 5 different timesteps $-380 \mathrm{Myr},-320 \mathrm{Myr}$, $-260 \mathrm{Myr},-190 \mathrm{Myr}$, and $-130 \mathrm{Myr}$, i.e. before ram pressure maximum. The evolution of the LRP1/2 and HRP1/2 models for a given disk-wind angle between the disk and the ram pressure wind are qualitatively similar. For timesteps $t<-150 \mathrm{Myr}$ the HI distribution of the different disk-wind angles are also similar. At $t=-380$ Myr there is more high surface density in the northwestern part of the galaxy, whereas we observe a low surfacedensity arm in the southeast. At $t=-320 \mathrm{Myr}$ the disk emission shows is approximately symmetric along the major axis and a low surface-density arm is formed east of the galaxy center. This snapshot corresponds to the LRP and HRP snapshots at $t=-120 \mathrm{Myr}$ (Figs. 13 and 14), because the ram pressure induced momentum transfer at the two respective timesteps is about the same (Fig. 10). At $t=-260 \mathrm{Myr}$ this arm is 

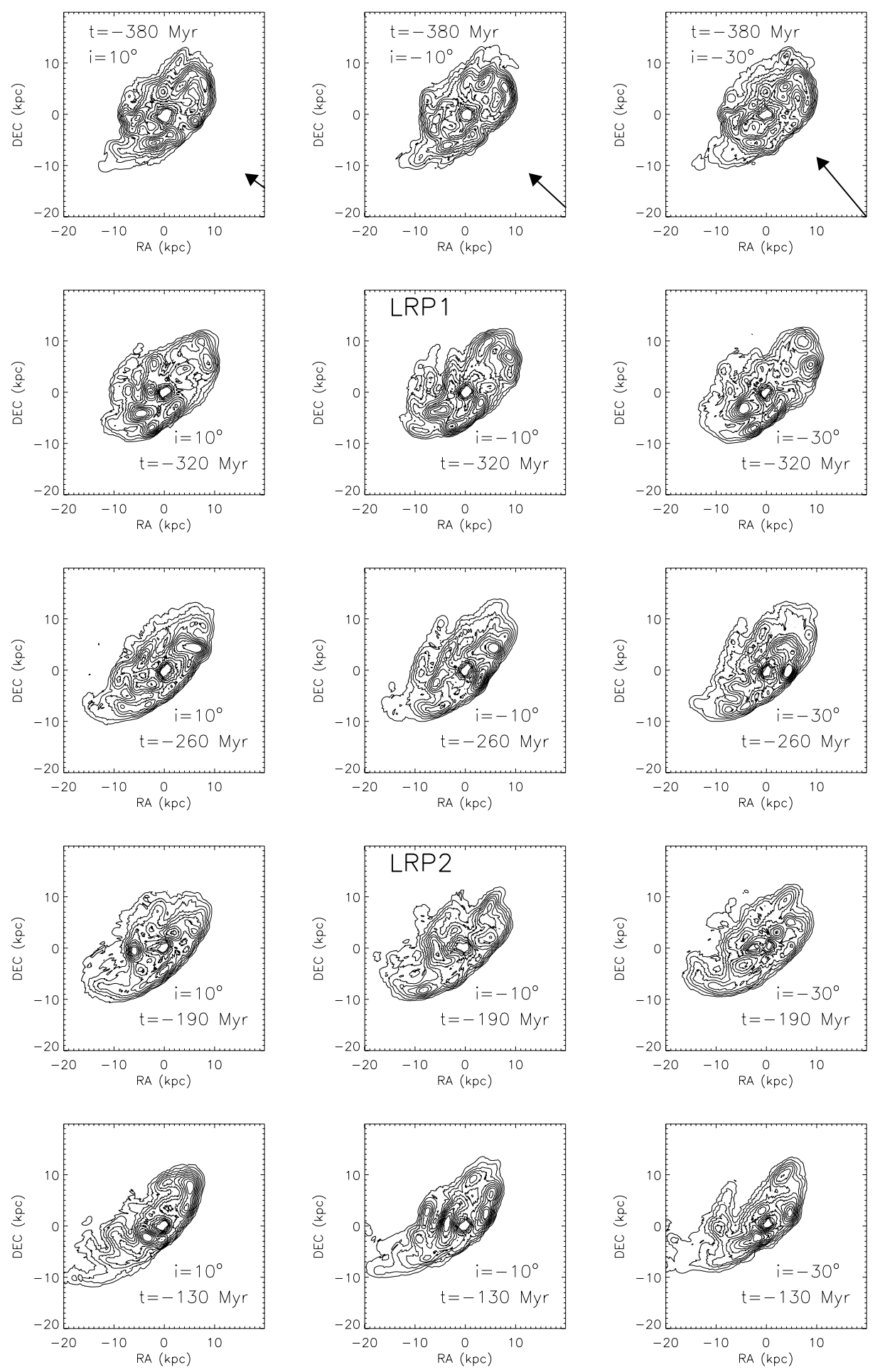

Fig. 15. Model snapshots of the gas column density for different simulations of the low ram pressure (LRP1/2) model with the large temporal ram pressure profile. Contours are the same as in Fig. 13. The ram pressure profile for all simulations is given by Eq. (2). The disk-wind angle $i$ between the orbital and the galaxy's disk plane and the timestep of the snapshot are varied.

located closer to the galaxy center and again a southeastern low surface-density arm is formed. A pronounced asymmetry along the minor axis is observed at $t=-190$ Myr with more emission in the compressed western part of the disk. In addition, we find a less pronounced asymmetry along the major axis with more emission in the southeastern part. At $t=-130 \mathrm{Myr}$ the gas in the western part of the disk is pushed to smaller radii and we observe a low surface-density arm in the southeast of the galaxy center. Based on this set of simulations, 4 snapshots with $i=-10^{\circ}$ reproduce our HI observations: LRP1 $t=-320 \mathrm{Myr}, \mathrm{LRP} 2-t=-190 \mathrm{Myr}, \mathrm{HRP} 1-t=-320 \mathrm{Myr}$, and HRP2 $-t=-190$ Myr. All 4 snapshots show a low surfacedensity gas located northeast of the galaxy center and an region of high surface-density gas at the opposite windward side. Only the snapshots at $t=-190 \mathrm{Myr}$ show an asymmetry along the major axis with a more extended emission to the southeast. 

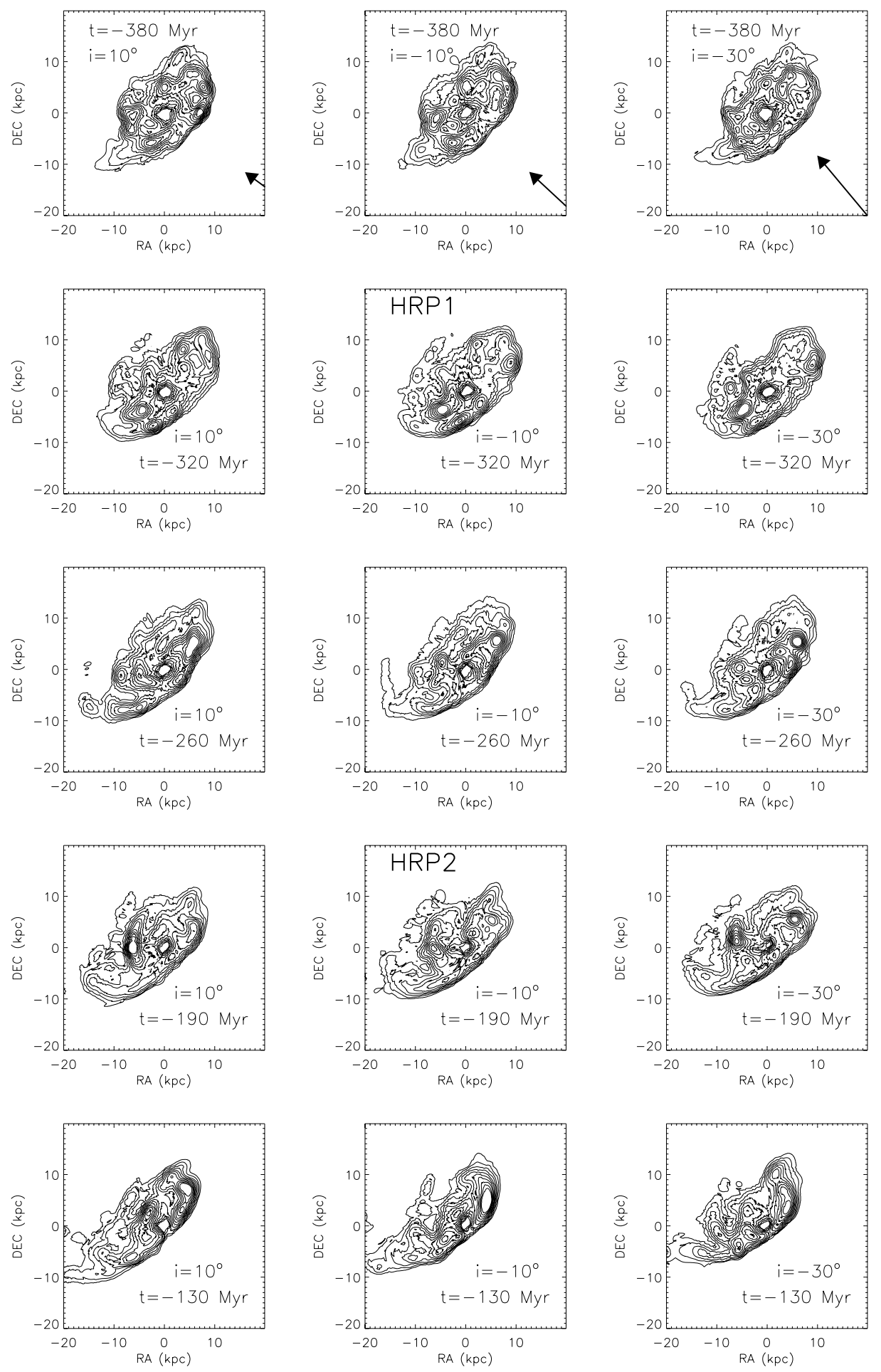

Fig. 16. Model snapshots of the gas column density for different simulations of the high ram pressure (HRP1/2) model with the large temporal ram pressure profile. Contours are the same as in Fig. 13. The ram pressure profile for all simulations is given by Eq. (2). The disk-wind angle $i$ between the orbital and the galaxy's disk plane and the timestep of the snapshot are varied.

The time evolutions of these simulations are shown in Fig. 17. Since ram pressure only affects the interstellar medium of the galaxy, the stellar disk stays symmetric during the whole simulation. The sky-projected wind direction is northeastward for the LRP1/2 and HRP1/2 model. As expected, the stripping radius of the HRP $1 / 2$ model is smaller than that of the LRP1/2 model, i.e. the galaxy in the HRP1/2 model loses more gas than that of the LRP1/2 model.

\subsection{Magnetic field evolution}

Otmianowska-Mazur \& Vollmer (2003) studied the evolution of the large-scale magnetic field during a ram pressure stripping event. They calculated the magnetic field structure by solving the induction equation on the velocity fields produced by the dynamical model. The polarized radio-continuum emission has been calculated by assuming a Gaussian spatial distribution of 
LRP1/2 MODEL
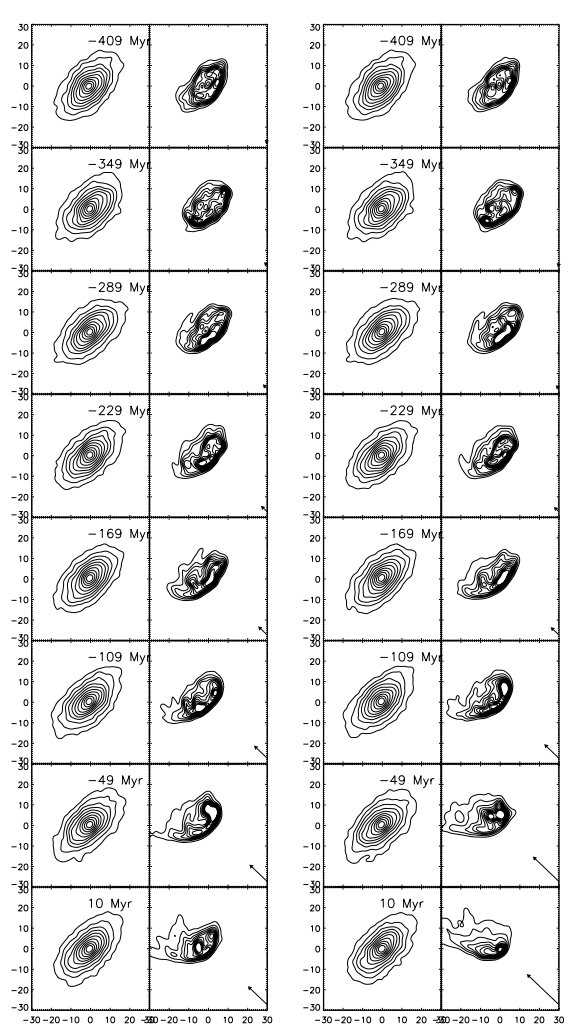

Fig. 17. Evolution of the model stellar (1st column) and gas disk (2nd column). The contours of the stellar distribution are logarithmic, those of the gas distribution are the same as in Fig. 13. The arrow indicates the direction of ram pressure, i.e. it is opposite to the galaxy's velocity vector, and its size is proportional to $\rho v_{\text {gal }}^{2}$. Maximum ram pressure occurs at $t=0 \mathrm{Myr}$. The timestep of each snapshot is marked in each panel showing the stellar disk.

relativistic electrons. This procedure allowed them to study the evolution of the observable polarized radio continuum emission during a ram pressure stripping event.

We apply the same procedure as Otmianowska-Mazur \& Vollmer (2003) to a similar ram pressure stripping event (Sect. 5). The Zeus3D code (Stone \& Norman 1992a,b) is used to solve the induction equation:

$\partial \boldsymbol{B} / \partial t=\operatorname{rot}(\boldsymbol{v} \times \boldsymbol{B})-\operatorname{rot}(\eta \operatorname{rot} \boldsymbol{B})$

where $\boldsymbol{B}$ is the magnetic induction, $\boldsymbol{v}$ is the large-scale velocity of the gas, and $\eta$ is the coefficient of a turbulent diffusion. We use a physical diffusion of $\eta=5 \times 10^{25} \mathrm{~cm}^{2} \mathrm{~s}^{-1}$ (Elstner et al. 2000). We do not implement any dynamo process. The initial magnetic field is purely toroidal with a strength of $10 \mu \mathrm{G}$.

The induction equation is solved on rectangular coordinates $(X Y Z)$. The number of grid points used is $215 \times 215 \times 91$ along the $X, Y$ and $Z$ axis, respectively with a grid size of $400 \mathrm{pc}$, resulting in a size of the modeled box of $86 \mathrm{kpc} \times$ $86 \mathrm{kpc} \times 36 \mathrm{kpc}$. Since the $N$-body code is discrete whereas the MHD code is using a grid, we have to interpolate the discrete velocities on the grid. This is done using a method known as "Kriging" with a density-dependent smoothing length (Isaaks \& Srivastava 1989). It turned out that we had to use a large smoothing length to suppress the noise in the velocity field of the outer disk, which is due to a small local particle density. In this way we avoid numerical artifacts in the velocity field at the outer disk (see Soida et al. 2006). As the spline interpolation used in Otmianowska-Mazur \& Vollmer (2003), this has the consequence that the velocity field at the edge of the gas distribution is more extended than the gas distribution itself. Since there are gradients in this velocity field due to differential rotation, induction leads to a magnetic field which extends beyond the edge of the gas distribution. This affects the polarized emission beyond the gas distribution, but not inside, which is what we are interested in.

The evolution of the polarized radio-continuum emission without Faraday rotation is presented in Fig. 18. The timesteps are the same as in Fig. 17. The disk rotates counter-clockwise. The gas surface density, which is smoothed to a resolution of $\sim 100 \mathrm{pc}$, is shown in greyscales, the polarized radio-continuum emission as contours, and the magnetic field vectors projected on the plane of the sky as lines. We assume a Gaussian distribution of relativistic electrons in $R$ and $z$ directions: $n_{\mathrm{rel}}=$ $n_{0} \exp \left(-\left(r / r_{\mathrm{R}}\right)^{2}\right) \exp \left(-\left(z / r_{z}\right)^{2}\right)$, where $r_{\mathrm{R}}=6.3 \mathrm{kpc}$ and $r_{z}=$ $1.0 \mathrm{kpc}$. This translates into a $F W H M$ of $5.2 \mathrm{kpc}$ and $0.83 \mathrm{kpc}$, respectively. By assuming this smooth distribution we imply no local equipartition between the energy densities of total cosmic rays and total magnetic field. The galaxy moves to the lower right corner, i.e. the ram pressure wind comes from this direction. Our grid size does not permit us to resolve the rotation in the inner part of the galaxy. The errors due to discretization lead to an artificial radial diffusion of the magnetic field out of the galactic disk. During the evolution without a significant external influence $(-380 \mathrm{Myr}<t<-290 \mathrm{Myr})$ the model distribution of polarized radio-continuum emission is approximately symmetric. Once ram pressure compresses the gas, an enhanced ridge of polarized emission appears in the southwest. At $t=-230 \mathrm{Myr}$ the internal magnetic field is lost due to the artificial diffusion, and only the compression induced ridge of polarized emission is visible. The evolution of polarized radio-continuum emission is very similar in the LRP1/2 and HRP1/2 models.

\section{Comparison between the models and observations}

In this section we compare the observed properties of the gas, star formation, and large-scale magnetic field of NGC 4501 with the properties of the simulated galaxies. Assuming a static intracluster medium, the projected direction of the ram pressure wind indicates the projected direction of the galaxy's motion within the intracluster medium. For the relevant simulations HRP1/2 and LRP1/2 with $i=-10^{\circ}$ this direction is southwest (Figs. 15 and 16). A time to peak ram pressure of $320 \mathrm{Myr}$ or $190 \mathrm{Myr}$ leads the galaxy to a distance of $0.4 \mathrm{Mpc}$ and $0.5 \mathrm{Mpc}$ from the cluster center at peak ram pressure assuming a linear orbit with a constant velocity (see Sect. 7). Projection effects will increase, orbital curvature and acceleration will decrease these values. An inspection of simulated galaxy orbits (Fig. 11) shows that the difference between the location at peak ram pressure and the extrapolated location assuming a linear orbit is $\sim 0.1 \mathrm{Mpc}$ for a timescale of $320 \mathrm{Myr}(\sim 0.03 \mathrm{Mpc}$ for a timescale of $190 \mathrm{Myr})$. Our adopted minimum distance scale is thus $0.3-0.4 \mathrm{Mpc}$. This distance is only compatible with the low ram pressure simulations LRP1/2 (see Sect. 5.2). Therefore, in the following we will only compare the LRP1/2 model to our multi-wavelength observations. 
LRP1/2 MODEL
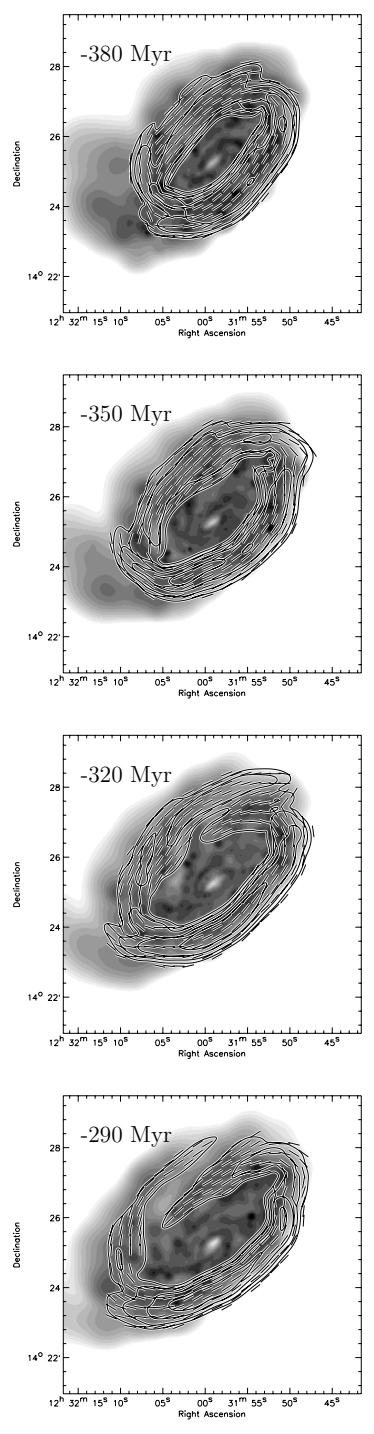
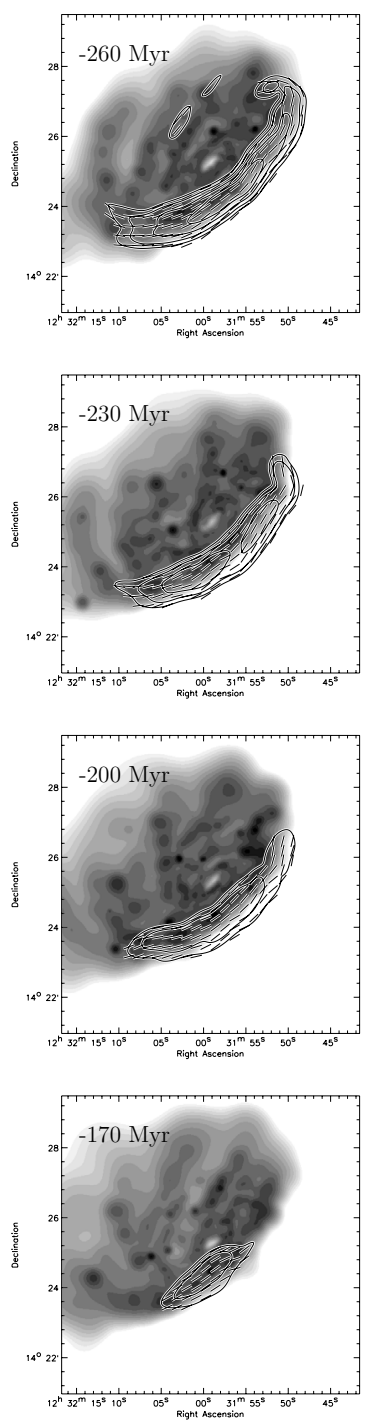

HRP1/2 MODEL
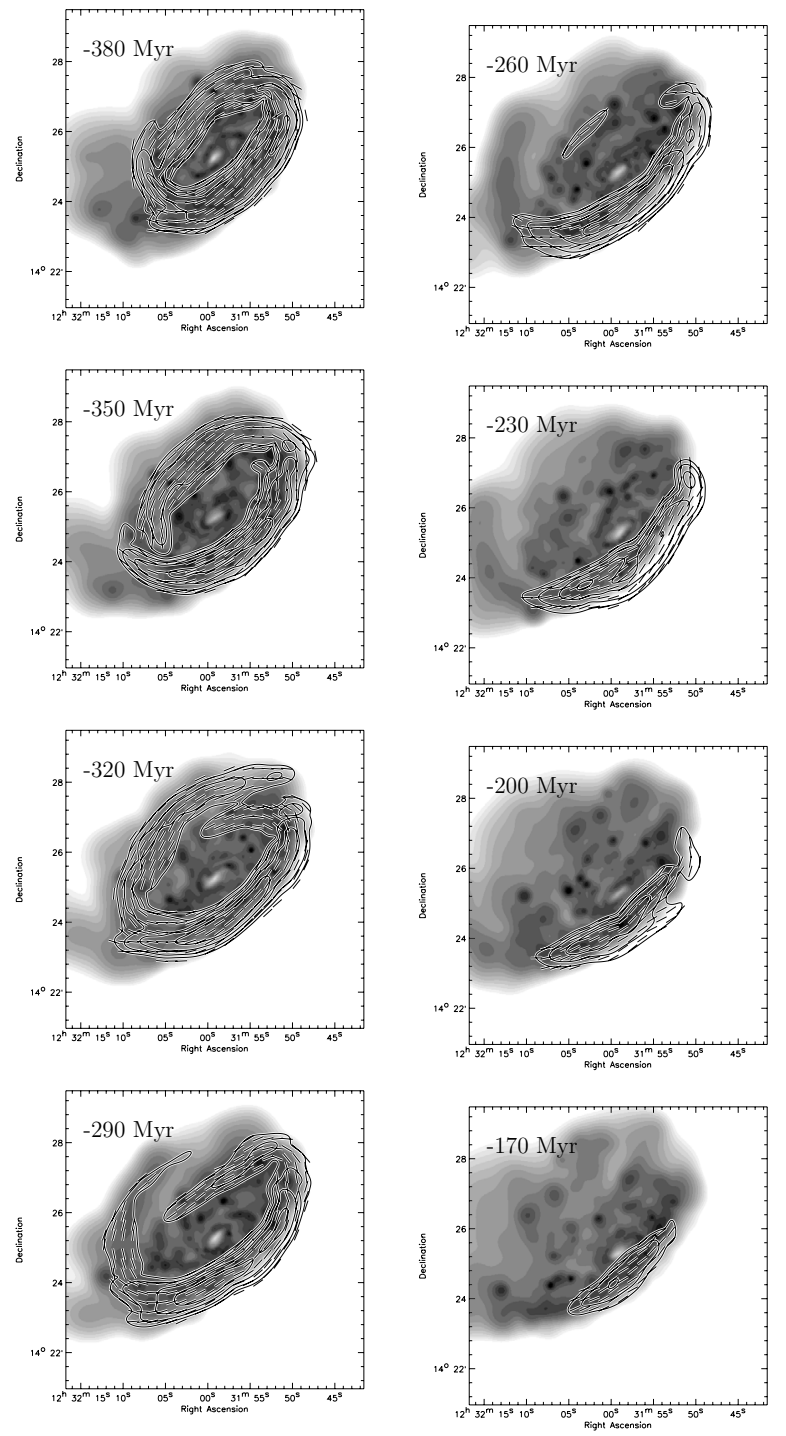

Fig. 18. Evolution of the polarized radio-continuum emission. Time steps are indicated in the upper left corner of each panel. The disk is seen with the position and inclination of NGC 4501 (Table 1) and rotates counter-clockwise. Greyscale: gas surface density in logarithmic scale. Contours: polarized radio-continuum emission. The magnetic field vectors are superimposed onto the gas surface density. The assumed beamsize for the polarized radio-continuum emission is $20^{\prime \prime}$.

\subsection{The gas moment maps}

As described in Sect. 3 the observed HI distribution follows one isophot contour of the optical image except in the northeast and the north. Our model snapshots LRP1 and LRP2 show the same qualitative behavior (Fig. 19). However, the LRP2 model gas distribution in the west is less extended than the observed HI distribution, i.e. the model gas is pushed to smaller galactic radii than it is observed. On the other hand, the observed asymmetry along the major axis is only reproduced by the LRP2 model.

Figure 20 compares the gas distribution, velocity field, and the distribution of the velocity dispersion of our HI observations with our simulations. Both models reproduce qualitatively the overall observed HI surface density distribution of the outer gas disk. Both models show an overdensity in the compressed southwestern region of the disk. Both models also show an extended low surface-density region in northeast, as it is observed. There are, however, quantitative differences: the northeastern low surface-density arm of the LRP1 model is (i) located more to the southeast and (ii) more detached from the disk, whereas that of the LRP2 model is (i) located more to the northwest and (ii) closer to the galaxy center than it is observed. The LRP2 gas distribution shows a local maximum $\sim 1^{\prime}$ southeast of the galaxy center. It is not excluded that a significant amount of this gas might be in molecular form.

Both models also reproduce qualitatively the overall gas kinematics. The two main characteristics of the observed HI velocity field, the earlier flattening of the rotation curve in the southeast and the regular velocity field in the extended low surface-density northeastern region indicating rotation, are well reproduced. This behavior is more pronounced in the LRP2 model snapshot. For a more detailed comparison we show position-velocity diagrams along the major axis, minor axis, and parallel to the minor axis $20^{\prime \prime}$ southeast from the galaxy center (Fig. 21). Whereas the observed rising rotation curve to the northwest is reproduced, the model rotation curve to the southeast decreases sightly, whereas the observed HI rotation curve stays constant. The model nicely reproduces the HI data for the 

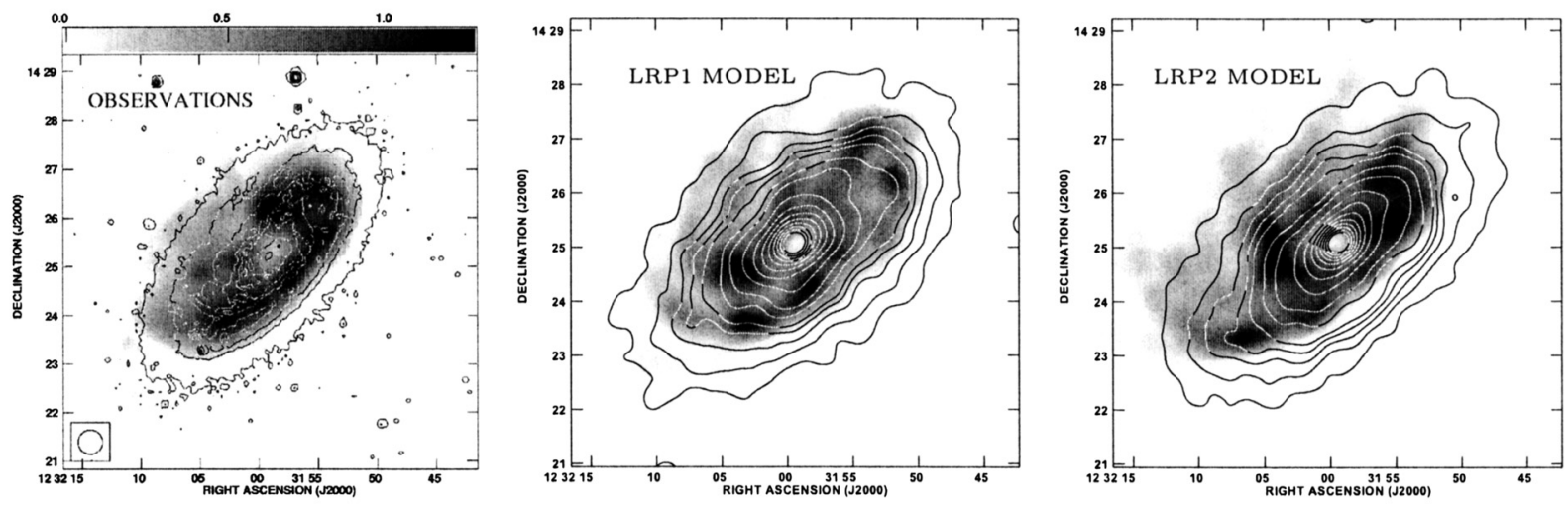

Fig. 19. Comparison between the gas surface density distribution (greyscale) and the stellar surface density distribution (contours). Left panel: $B$ band contours on HI surface density distribution (resolution: $30^{\prime \prime}$ ). In all figures the greyscales use the full dynamic ranges of the gas distribution.
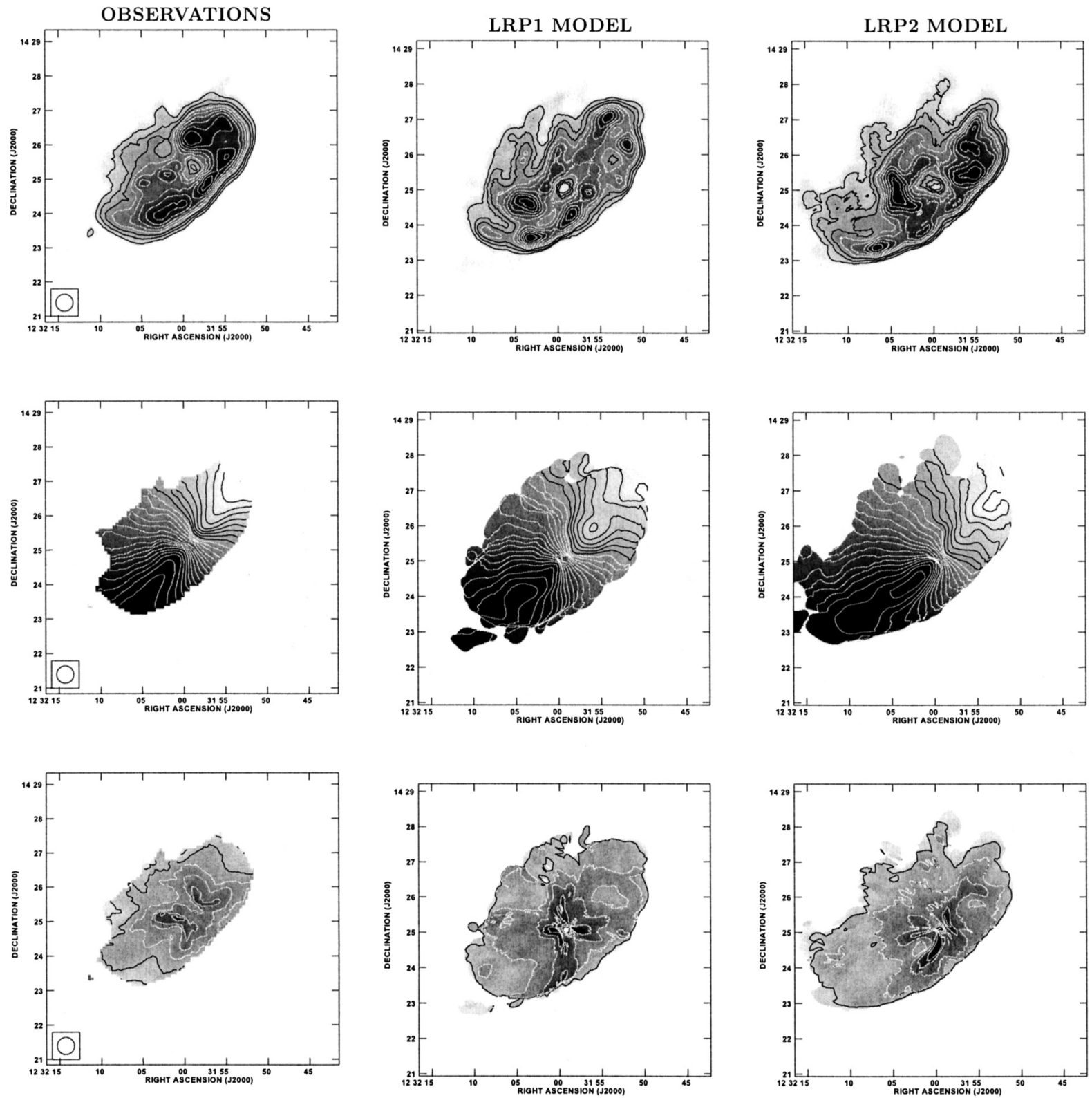

Fig. 20. Comparison between the observed $\mathrm{HI}$ and simulated gas moment maps. Upper row: gas distribution; middle row: velocity field; lower row: velocity dispersion. 

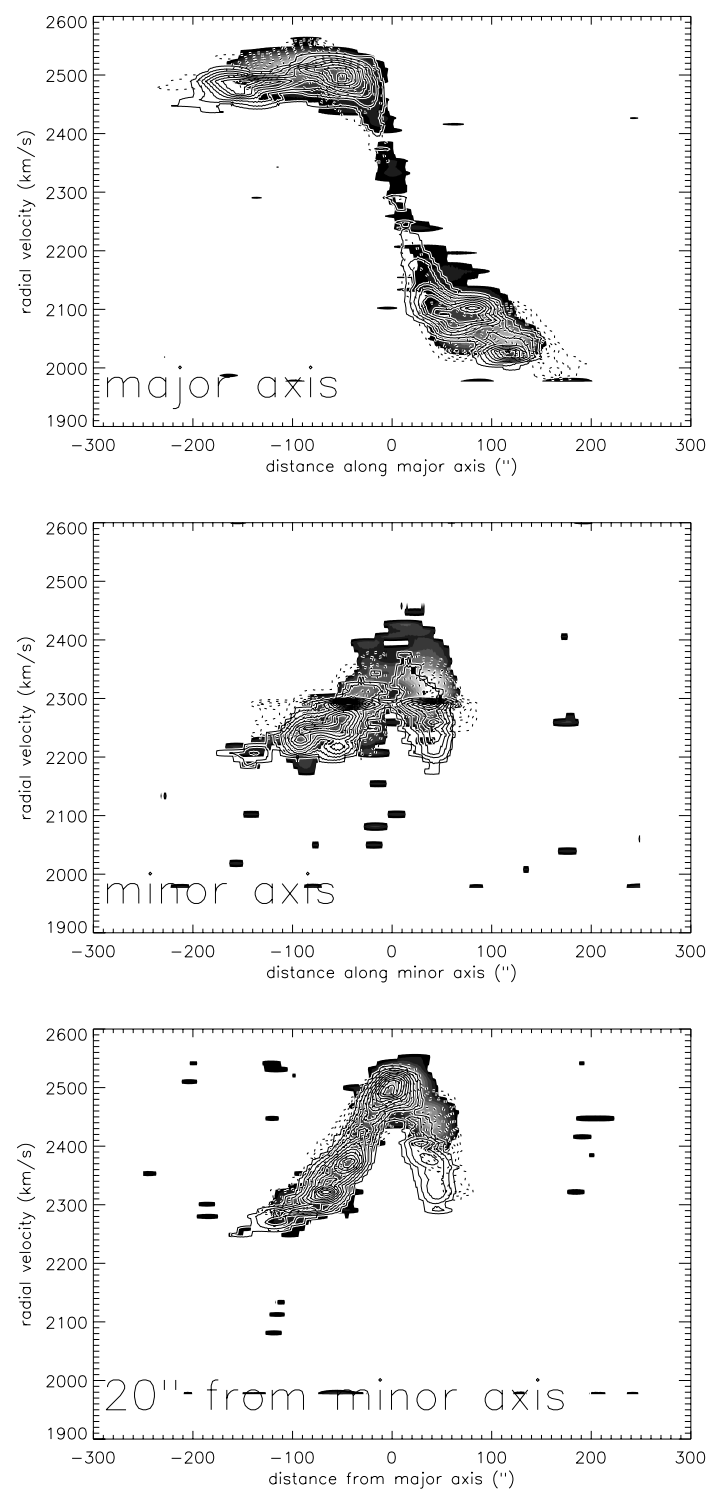

Fig. 21. Comparison between observed HI (greyscale) and simulated position-velocity diagrams for the LRP1 (dotted line) and LRP2 model (solid line). Upper panel: cut along the major axis, northwest is to the right; middle panel: cut along the minor axis, northeast is to the left; lower panel: cut parallel to the minor axis $20^{\prime \prime}$ southeast from the galaxy center, northeast is to the left.

cuts along and parallel to the minor axis. The LRP1 model distribution of the dispersion velocity reasonably agree with the HI observations. That of the LRP2 model shows an asymmetry along the major axis, with a larger velocity dispersion to the southeast due to radial velocities induced by ram pressure compression, which is not observed.

\subsection{The star formation distribution}

The collisional component of the model consists of 20000 gas clouds of different mass that can have inelastic collisions. For particles of equal size the collision rate, i.e. the number of collisions per unit time, is $\mathrm{d} N / \mathrm{d} t=n \sigma v$ where $n$ is the particle volume density, $\sigma$ the cross section, and $v$ the particle velocity. The collisional timescale is $t_{\text {coll }}=(n \sigma v)^{-1}$. The mass involved in collisions per unit time and unit area is thus $\dot{\Sigma}=\Sigma n \sigma v$, where $\Sigma$ is the surface density. We now assume that the star formation $\dot{\Sigma}_{*}$ rate is proportional to the cloud collision rate $\dot{\Sigma}_{*} \propto \dot{\Sigma}$. In a turbulent selfgravitating gas disk in hydrostatic equilibrium $n \propto \Omega^{2}$ and $\Sigma \propto \Omega$, where $\Omega$ is the angular velocity (Vollmer \& Beckert 2002, 2003). Thus, we obtain $\dot{\Sigma}_{*} \propto \Sigma^{1.5}$ which is close to the observed Schmidt law (see, e.g. Wong \& Blitz 2002). To compare the model star formation rates with $\mathrm{H} \alpha$ observations we gather all collisions up to $10 \mathrm{Myr}$ before the timestep of interest. The resulting model star formation rates are shown in Fig. 22. These snapshots can be directly compared to Fig. 7. Both model snapshots show a ring of star formation in the inner $5 \mathrm{kpc}$ and enhanced star formation along the compressed ridge of high column density gas at the southwestern windward side. The northwestern $\mathrm{H} \alpha$ spiral arm is less prominent in the model. The observed lack of star formation at $\sim 1^{\prime}$ east from the galaxy center is only present in the LRP 2 model. This is directly linked to the discrepancy of the observed and simulated gas surface density at this location discussed in Sect. 6.1. The underlying gas cloud distribution of the LRP2 model snapshot is shown in Fig. 23. The observed faint outer $\mathrm{H} \alpha$ arm in the west of the galactic disk is not present in our model where the star formation peaks together with the gas distribution. However, we observe a qualitatively similar behavior of the LRP2 model star formation distribution southwest from the galaxy center: there are two distinct arms with a clear gap between them. The external arm is due to ram pressure compression, the internal arm is a spiral arm. Since the $\mathrm{H} \alpha$ emission distribution also shows a double arm structure in the southwest (Fig. 7), we suggest that the southern part of the compressed HI ridge consists of two distinct narrow gas arms which are smeared out by the $30^{\prime \prime}$ resolution of the HI observations. We thus predict that future high resolution HI observations detect these two arms.

\subsection{The polarized radio-continuum emission}

The comparison between the observed and simulated gas surface density distribution, the polarized radio-continuum emission, and the sky-projected vectors of the large-scale magnetic field is shown in Fig. 24. As explained in Sect. 5.3 we lose the large-scale magnetic field in the inner disk due to numerical diffusion. Moreover, the observed polarized emission in the inner disk could be due to regular fields or compressed random fields due to dynamo action or compressed fields due to density waves, which are not part of the model. The model polarized emission is due to an enhancement of the large-scale magnetic field by shear and radial motions (induction equation) which are well present in the model velocity fields. Therefore, while the loss of magnetic field energy by diffusion is a numerical effect, the gain of magnetic field energy, and thus the enhancement of polarized radio-continuum emission, is real. We successfully reproduce the enhanced polarized radio-continuum distribution at the edge of the compression region. As observed the model polarized emission peaks in front of the HI emission for the LRP1 model. However, the south-eastern maximum of the LRP2 model is located at the backside of the HI maximum. The magnetic field vectors are parallel to this edge for the LRP1, in agreement with observations, whereas they deviate from this direction at the location of the southeastern maximum of the LRP2 model.

\section{Discussion}

The first set of simulations with the temporal ram pressure profiles used in Vollmer et al. (2001, 2005, 2006) qualitatively reproduce the observed HI surface density distribution (LRP of Fig. 13 and HRP of Fig. 14). However, the deduced timescale 

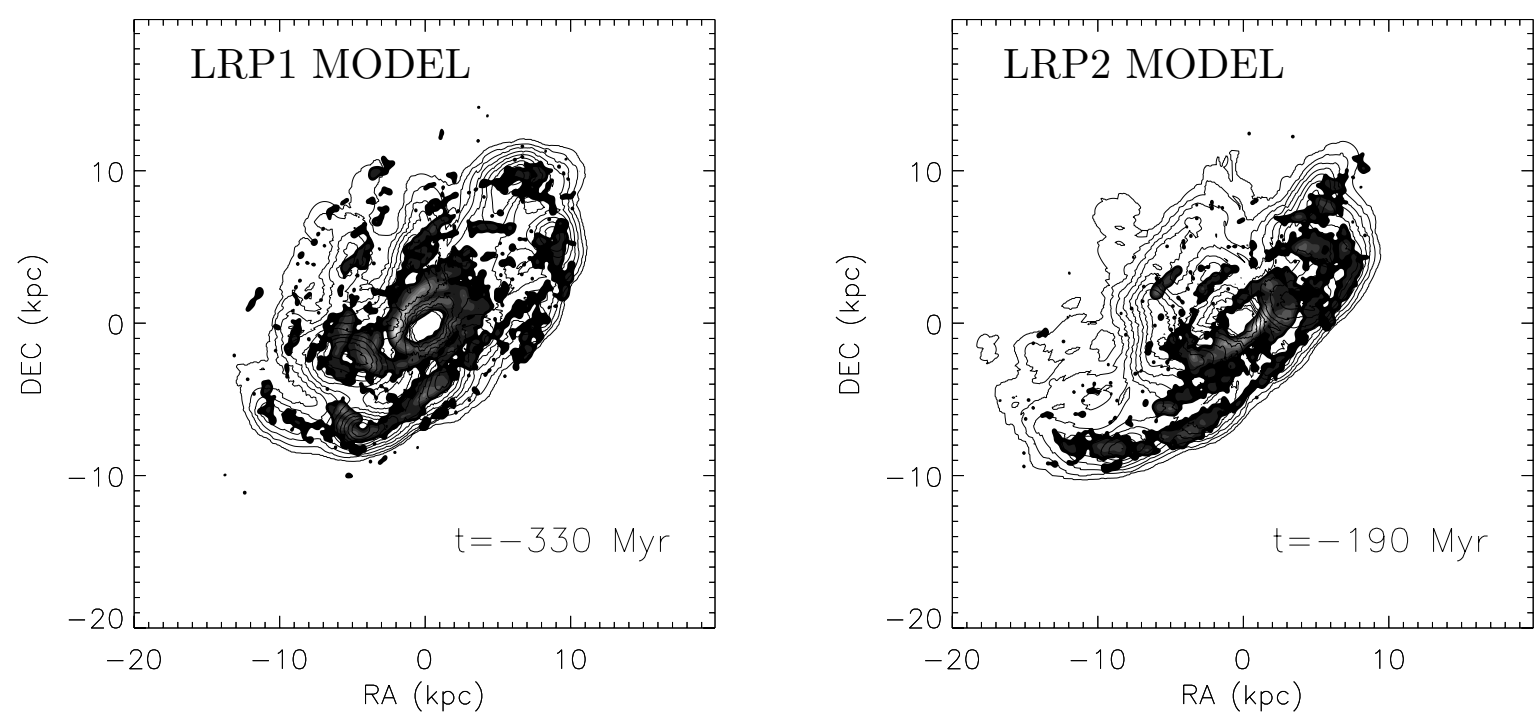

Fig. 22. Greyscale: model star formation distribution. Contours: model gas distribution. The timestep is indicated in the lower right corner of each panel.

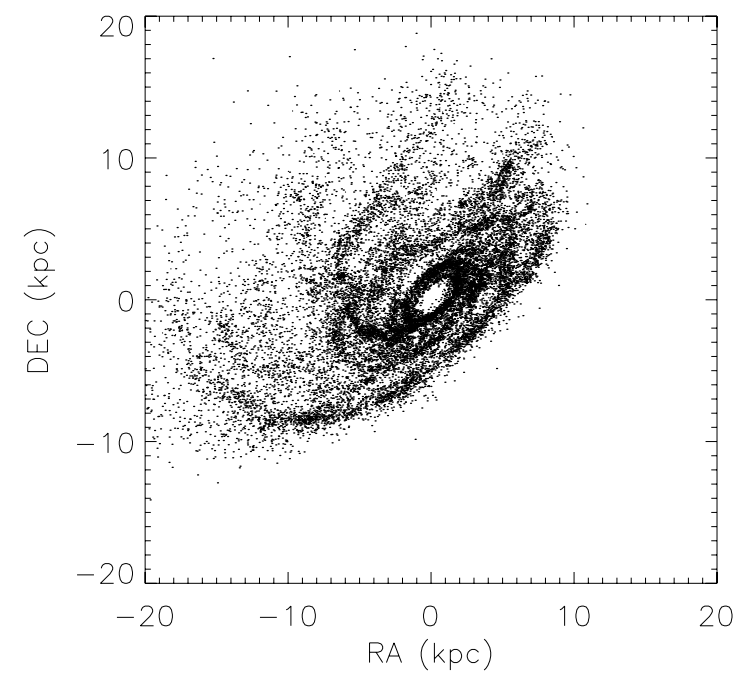

Fig. 23. Model cloud distribution at $t=-190 \mathrm{Myr}$ of the LRP2 snapshot.

to peak ram pressure of $120 \mathrm{Myr}$ is too short for the galaxy to reach a position near enough to the cluster center where the expected ram pressure corresponds to the model peak ram pressure. Therefore, we made a second set of simulations where we doubled the widths of the temporal ram pressure profiles. Since the inferred motion of the galaxy within the intracluster medium does not permit it to reach a position where the peak ram pressure is strong (as for NGC 4438 where $p_{\max }=$ $\left.5000 \mathrm{~cm}^{-3}\left(\mathrm{~km} \mathrm{~s}^{-1}\right)^{2}, \mathrm{HRP} 1 / 2\right)$, we prefer the simulations with moderately strong peak ram pressure $\left(p_{\max }=2000 \mathrm{~cm}^{-3}\right.$ $\left.\left(\mathrm{km} \mathrm{s}^{-1}\right)^{2}, \mathrm{LRP} 1 / 2\right)$. These dynamical simulations qualitatively reproduce the observed HI surface density distribution and kinematics (Fig. 20), the observed massive star formation distribution (traced by $\mathrm{H} \alpha$ emission; Fig. 22), and the $6 \mathrm{~cm}$ polarized radio continuum emission distribution (Fig. 24).

Within the LRP1/2 model the current ram pressure on NGC 4501 is $400 \mathrm{~cm}^{-3}\left(\mathrm{~km} \mathrm{~s}^{-1}\right)^{2}$ (LRP1 model) and $800 \mathrm{~cm}^{-3}\left(\mathrm{~km} \mathrm{~s}^{-1}\right)^{2}$ (LRP2 model). The direction of the model galaxy's three dimensional velocity vector is $(0.48,-0.44,0.76)$
( $x$-axis: RA, positive to the west; $y$-axis: Dec, positive to the north; $z$-axis: line-of-sight, positive for the far side). Based on this geometry derived from our simulations, NGC 4501's orbital velocity is $1500 \mathrm{~km} \mathrm{~s}^{-1}$ for both model snapshots LRP1 and LRP2. A simplified Gunn \& Gott criterion (Gunn \& Gott 1972) yields

$\Sigma_{\mathrm{ISM}} \frac{v_{\mathrm{rot}}^{2}}{R}=\rho_{\mathrm{ICM}} v_{\mathrm{gal}}^{2}$,

where $R$ is the stripping radius, $\Sigma_{\text {ISM }}$ the HI column density, $v_{\text {rot }}$ the rotation velocity, and $v_{\text {gas }}$ the orbital velocity of NGC 4501 . This estimate assumes a smooth and static intracluster medium. Taking an HI column density of $\Sigma_{\text {ISM }}=10^{20} \mathrm{~cm}^{-2}$ and a stripping radius of $12.5 \mathrm{kpc}$, we derive an intracluster medium density of $\rho_{\mathrm{ICM}}=10^{-4} \mathrm{~cm}^{-3}$. This is consistent with the intracluster medium density at the projected location of NGC 4501 derived from X-ray observations under the assumption of hydrostatic equilibrium $\rho_{\mathrm{ICM}}=7 \times 10^{-5} \mathrm{~cm}^{-3}$ (Schindler et al. 1999).

The LRP1 model better reproduces (i) the outer edge of the HI distribution compared to the optical isophots (Fig. 19), (ii) the symmetric distribution of the velocity dispersion (Fig. 20), (iii) the distribution of the polarized radio-continuum emission (Fig. 24), and (iv) the galaxy has more time to approach the cluster center (320 Myr to peak ram pressure). On the other hand, the LRP2 model better reproduces (i) the asymmetric HI distribution along the major axis with a larger extent to the southeast, (ii) the steepening of the rotation curve to the northwest and its flattening to the southeast (Fig. 21), and (iii) the gap between two arms of star formation at the western edge of the galactic disk (Fig. 22). The timescale to ram pressure maximum of $190 \mathrm{Myr}$ is smaller than that of the LRP1 model, but still sufficient to reach a position in the cluster where the model peak ram pressure can be reached. Based on these arguments we regard the timescale of 320 Myr to peak ram pressure as an upper limit.

Figure 25 shows the linear extrapolation of NGC 4501's probable trajectory. With a radial velocity with respect to the Virgo cluster mean of $1130 \mathrm{~km} \mathrm{~s}^{-1}$ a velocity within the sky plane of $\sim 1000 \mathrm{~km} \mathrm{~s}^{-1}$ is inferred. Thus, the galaxy travels 0.33/0.19 Mpc from its present location within 320/190 Myr. At maximum ram pressure the galaxy's trajectory is perpendicular to its distance vector to $M 87$. The projected angles between the 

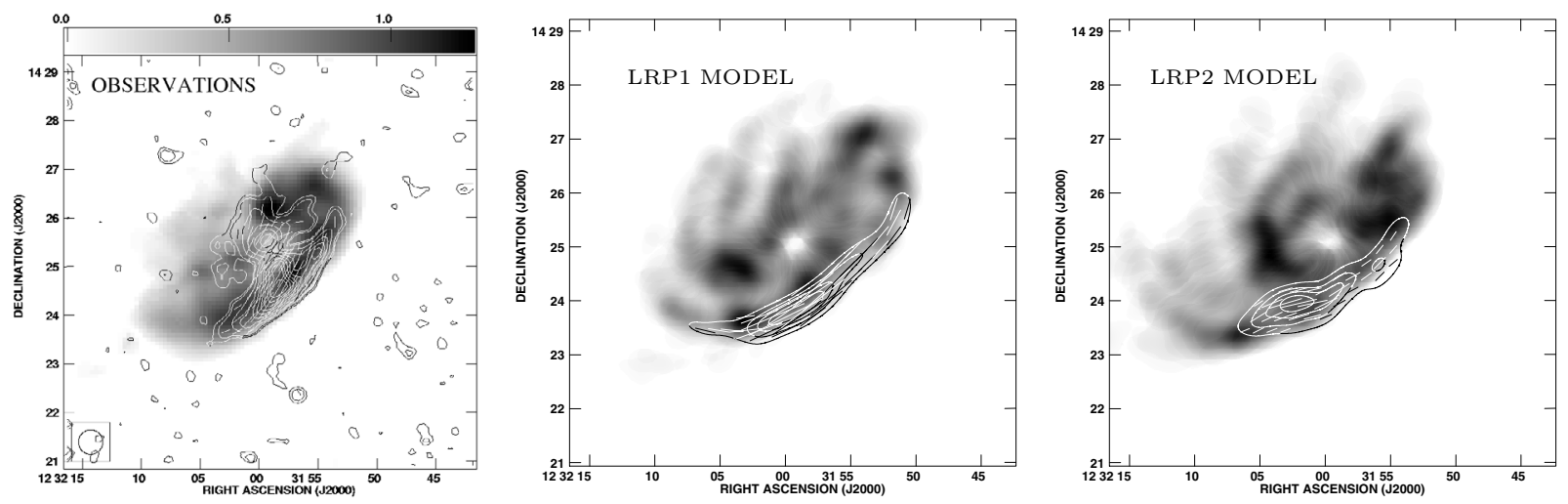

Fig. 24. Comparison between the observed and simulated gas surface density distribution (greyscale; resolution: 30"), the polarized radiocontinuum emission (contours; resolution: 18"), and the sky-projected vectors of the large-scale magnetic field.

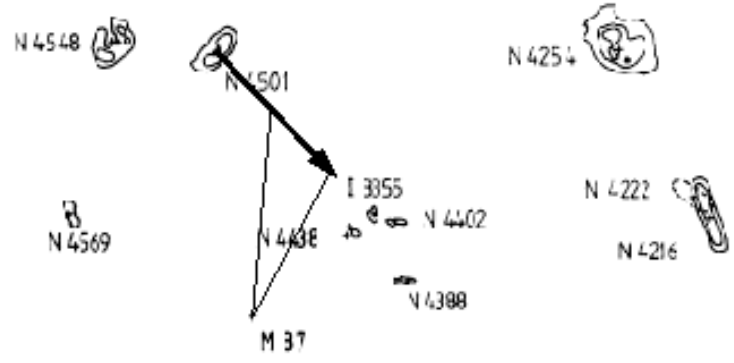

$\$+0 \times 45 \%$

Fig. 25. Sketch of the linear extrapolation of NGC 4501's probable trajectory within the Virgo cluster. Contours show the HI surface density of the large spiral galaxies around the cluster center (M 87; from Cayatte et al. 1990). The projected distance between NGC 4501 and M 87 is $2^{\circ}$ $(0.6 \mathrm{Mpc})$. The two lines between M 87 and the trajectory correspond to the extrapolated galaxy's projected distance at peak ram pressure for the LRP2/HRP2 snapshots (the galaxy is observed 190 Myr before peak ram pressure) and the LRP1/HRP1 snapshots (the galaxy is observed $320 \mathrm{Myr}$ before peak ram pressure).

distance vector to M 87 and the galaxy's trajectory are $112^{\circ} / 125^{\circ}$ and the projected distances to $\mathrm{M} 87$ are $0.3 \mathrm{Mpc} / 0.48 \mathrm{Mpc}$, respectively. This translates into a three dimensional distance to M 87 of $0.39 \mathrm{Mpc}$ and $0.54 \mathrm{Mpc}$ at maximum ram pressure, respectively. The line-of-sight distance from M 87 at this moment is then $0.14 \mathrm{Mpc}$ and $0.27 \mathrm{Mpc}$, respectively. The present lineof-sight distance of NGC 4501 is $0.11 \mathrm{Mpc}$ in front of M 87 for the LRP1 model $(\Delta t=-320 \mathrm{Myr})$ and $0.13 \mathrm{Mpc}$ behind $\mathrm{M} 87$ for the LRP2 model ( $\Delta t=-190 \mathrm{Myr})$.

This overall picture (see also Table 3 ) makes us conclude that the outer western ridge of star formation is induced by nearly edge-on ram pressure stripping $\left(i \sim 10^{\circ}\right)$. A comparable case is NGC 4654 (Vollmer et al. 2003), where nearly edge-on ram pressure stripping $\left(i \sim 0^{\circ}\right)$ leads to an enhanced northwestern $\mathrm{H} \alpha$ emission in the compression region. This hypothesis can be verified by the determination of the star formation history from multiband photometry including UV emission and optical spectra. If our scenario is correct we expect a recent small starburst that created the western outer $\mathrm{H} \alpha$ arm.

\section{Conclusions}

VIVA HI observations of the Virgo spiral galaxy NGC 4501 are presented. The outer edge of the HI distribution follows one optical isophot contour except in the northeast, where a region of low surface-density gas is discovered. Whereas the $B$ band image is asymmetric, the lowest $B$ band isophot contour and the $H$ band isophot contours are symmetric. The observed southwestern ridge of $6 \mathrm{~cm}$ polarized radio-continuum emission (Vollmer et al. 2007) is located at the outer edge of the HI distribution. Both show the same rapid increase at the edge, which is expected in a ram pressure scenario. Along the gas ridge, a faint $\mathrm{H} \alpha$ emission ridge is present. The maximum of the polarized radio-continuum emission are located within the gap between the $\mathrm{H} \alpha$ emission of the spiral arms and the faint emission ridge.

We compare these observational characteristics with detailed dynamical models including MHD calculations. Models with different galaxy orbits (high and low ram pressure) and diskwind angles between the disk and the orbital plane are presented. Model snapshots at different times from peak ram pressure are compared to the multi-frequency observations. For the first time we present model maps of massive star formation. We qualitatively reproduce the gas distribution, kinematics, star formation distribution, and polarized radio-continuum emission distribution. We conclude that ram pressure stripping can account for the main characteristic. The western ridge of enhanced gas surface density and enhanced polarized radio-continuum emission is due to ram pressure compression. Assuming radial orbits in a static intracluster medium of the Virgo cluster (Vollmer et al. 2001), NGC 4501 will have its nearest approach to the cluster core, i.e. peak ram pressure, in $\sim 200-300 \mathrm{Myr}$ (Fig. 9). It is argued that the faint western $\mathrm{H} \alpha$ emission ridge is induced by nearly edge-on ram pressure stripping.

Acknowledgements. B.V. would like to thank the MPIfR (P. Reich) for computational support. This research has made use of the GOLD Mine Database. This work was supported by Polish-French (ASTRO-LEA-PF) cooperation program, and by Polish Ministry of Sciences grant PB 378/P03/28/2005 and PB 2693/H03/2006/31. We would like to thank the referee for his stimulating comments.

\section{References}

Beck, R. 2005, in Cosmic Magnetic Fields, ed. R. Wielebinski, \& R. Beck (Berlin: Springer), 41

Beck, R., Fletcher, A., Shukurov, A., et al. 2005, A\&A, 444, 739

Böhringer, H., Neumann, D. M., Schindler, S., \& Huchra, J. P. 1997, ApJ, 485, 439

Boulares, A., \& Cox, D. P. 1990, ApJ, 365, 544

Cayatte, V., van Gorkom, J. H., Balkowski, C., \& Kotanyi, C. 1990, AJ, 100, 604 
Cayatte, V., Kotanyi, C., Balkowski, C., \& van Gorkom, J. H. 1994, AJ, 107, 1003

Chamaraux, P., Balkowski, C., \& Gérard, E. 1980, A\&A, 83, 38

Chung, A., van Gorkom, J. H., Kenney, J. D. P., \& Vollmer, B. 2007, ApJ, 659, L115

de Vaucouleurs, G., de Vaucouleurs, A., Corwin, H. G., et al. 1991, Third Reference Catalogue of Bright Galaxies (New York: Springer) (RC3)

Elmegreen, B. G., \& Falgaron, E. 1996, ApJ, 471, 816

Elstner, D., Otmianowska-Mazur, K., von Linden, S., \& Urbanik, M. 2000, A\&A, 357, 129

Gavazzi, G., Boselli, A., Donati, A., et al. 2003, A\&A, 400, 451

Giovanelli, R., \& Haynes, M. P. 1983, AJ, 88, 881

Guhathakurta, P., van Gorkom, J. H., Kotanyi, C. G., \& Balkowski, C. 1988, AJ, 96,85

Gunn, J. E., \& Gott, J. R. 1972, ApJ, 176, 1

Harnett, J., Ehle, M., Fletcher, A., et al. 2004, A\&A, 421, 571

Hummel, E., \& Beck, R. 1995, A\&A, 303, 691

Isaaks, E. H., \& Srivastava, R. 1989, An Introduction to Applied Geostatistics (Oxford University Press), Chapter 12

Nulsen, P. E. J. 1982, MNRAS, 198, 1007
Otmianowska-Mazur, K, \& Vollmer, B. 2003, A\&A, 402, 879

Schindler, S., Binggeli, B., \& Böhringer, H. 1999, A\&A, 343, 420

Soida, M., Urbanik, M., \& Beck, R. 1996, A\&A, 312, 409

Soida, M., Urbanik, M., Beck, R., Wielebinski, R., \& Balkowski, C. 2001, A\&A, 378,40

Soida, M., Otmianowska-Mazur, K., Chyzy, K., \& Vollmer, B. 2006, A\&A, 458, 727

Springel, V., Yoshida, N., \& White, S. D. M. 2001, NewA, 6, 79

Stone J. M., \& Norman, M. L. 1992a, ApJS, 80, 791

Stone, J. M., \& Norman, M. L. 1992b, ApJS, 80, 791

Vollmer, B. 2003, A\&A, 398, 525

Vollmer, B., \& Beckert, T. 2002, A\&A, 382, 872

Vollmer, B., \& Beckert, T. 2003, A\&A, 404, 21

Vollmer, B., \& Huchtmeier, H. 2007, A\&A, 462, 93

Vollmer, B., Cayatte, V., Balkowski, C., \& Duschl, W. J. 2001, ApJ, 561, 708

Vollmer, B., Braine, J., Combes, F., \& Sofue, Y. 2005, A\&A, 441, 473

Vollmer, B., Soida, M., Otmianowska-Mazur, K., et al. 2006, A\&A, 453, 883

Vollmer, B., Soida, M., Beck, R., et al. 2007, A\&A, 464, L37

Wiegel, W. 1994, Diploma Thesis, University of Heidelberg

Wong, T., \& Blitz, L. 2002, ApJ, 569, 157 